\title{
'Nature is the Realisation of the Simplest Conceivable Mathematical Ideas': Einstein and the Canon of Mathematical Simplicity
}

\author{
John D. Norton*
}

\begin{abstract}
Einstein proclaimed that we could discover true laws of nature by seeking those with the simplest mathematical formulation. He came to this viewpoint later in his life. In his early years and work he was quite hostile to this idea. Einstein did not develop his later Platonism from a priori reasoning or aesthetic considerations. He learned the canon of mathematical simplicity from his own experiences in the discovery of new theories, most importantly, his discovery of general relativity. Through his neglect of the canon, he realised that he delayed the completion of general relativity by three years and nearly lost priority in discovery of its gravitational field equations. (C) 2000 Elsevier Science Ltd. All rights reserved.
\end{abstract}

Keywords: Einstein; Simplicity; Platonism; Relativity; Gravitation; Mathematics

\section{Introduction}

The account is developed in Sections 1-6 with a minimum of mathematical technicalities. Readers interested in them will find them exiled in Appendices $A-C$ at the end of the paper and also a brief critique of Einstein's view in Appendix D.

On 10 June 1933, Einstein gave his Herbert Spenser Lecture 'On the Methods of Theoretical Physics' at the University of Oxford (Einstein, 1933a). He lectured

* Department of History and Philosophy of Science, University of Pittsburgh, Pittsburgh PA 15260, U.S.A. (e-mail: jdnorton+@pitt.edu). 
for the first time in English, reading from a prepared translation. ${ }^{1}$ The lecture was to describe the methods used to discover theories in physics. It began with a self-mocking warning, perhaps because he sensed the account he was about to give was shocking: If you want to find out anything from the theoretical physicists about the methods they use, I advise you to stick closely to one principle: do not listen to their words, fix your attention on their deeds'. As the lecture developed it was not immediately apparent why Einstein should have entered into such an experiment in irony. He proceeded to a safe, empiricist disclaimer on the limited role of pure logic in science: 'Pure logical thinking cannot yield us any knowledge of the empirical world; all knowledge of reality starts from experience and ends with it'. This was followed by a more adventurous claim: while the fundamental concepts and postulates of our theories must accommodate experience, they are otherwise 'free inventions of the human intellect'. This freedom, he continued, had not been recognised in earlier centuries when natural philosophers erroneously believed that these concepts and postulates could be deduced from experience. So how are we to find them? ${ }^{2}$ That seemingly innocent question brought forth a manifesto so grandiose that one would dismiss it as empty posturing were it not the mature reflections of that scientist of the modern age, whose achievements had made his name synonymous with genius:

If, then, it is true that the axiomatic basis of theoretical physics cannot be extracted from experience but must be freely invented, can we ever hope to find the right way? Nay, more, has this right way any existence outside our illusions? Can we hope to be guided safely by experience at all when there exist theories (such as classical mechanics) which to a large extent do justice to experience, without getting to the root of the matter? I answer without hesitation that there is, in my opinion, a right way, and that we are capable of finding it. Our experience hitherto justifies us in believing that nature is the realisation of the simplest conceivable mathematical ideas. I am convinced that we can discover by means of purely mathematical constructions the concepts and the laws connecting them with each

\footnotetext{
${ }^{1}$ According to Fölsing (1997, p. 673).

${ }^{2}$ One might wonder if Einstein's position is consistent if he can portray this as a difficult task with a right answer. There is no problem arriving at free inventions - one just invents them! What makes the invention process difficult, however, is that the results are only admissible if they can accommodate a broad range of our experience. Finding inventions that do this is far from automatic. Moreover, while he portrayed the concepts and postulates as free inventions as a matter of general principle, he also urged that in practical terms there turns out to be one theoretical system that does the job best, much as we might propose any word to solve a word puzzle but only ' $[\ldots]$ one word which solves the puzzle in all its parts' (Einstein, 1936, pp. 294-295; Einstein's emphasis). Consider also Einstein's (1918a, p. 226) earlier remark that 'in practice the world of phenomena uniquely determines the theoretical system'. The right method leads us to this system. That this system is not to be thought of instrumentally is made clear by his elaboration of the heuristic of mathematical simplicity in the Herbert Spenser Lecture when he explains (p. 275): 'In the limited number of the mathematically existent simple field types, and the simple equations possible between them, lies the theorist's hope of grasping the real in all its depth'. His goal is not just a good instrument for calculating experience but 'the real in all its depth'.
} 
other, which furnish the key to the understanding of natural phenomena. Experience may suggest the appropriate mathematical concepts, but they most certainly cannot be deduced from it. Experience remains, of course, the sole criterion of the physical utility of a mathematical construction. But the creative principle resides in mathematics. In a certain sense, therefore, I hold it true that pure thought can grasp reality, as the ancients dreamed.

To remove any doubt that Einstein had a quite concrete understanding of this 'right method', he proceeded to show how it leads directly to the two most successful theories of his time outside the quantum domain, Maxwell's electrodynamics and his own general theory of relativity:

In order to justify this confidence, I am compelled to make use of a mathematical concept. The physical world is represented as a four-dimensional continuum. If I assume a Riemannian metric in it and ask what are the simplest laws which such a metric can satisfy, I arrive at the relativistic theory of gravitation in empty space. If in that space I assume a vector-field or an anti-symmetrical tensor field which can be derived from it, and ask what are the simplest laws which such a field can satisfy, I arrive at Maxwell's equations for empty space.

My purpose in this paper is to reveal where Einstein found his confidence in the supreme heuristic power of mathematical simplicity. It was not an a priori aesthetic judgement or the outpourings of a physicist eager to extract impressive philosophical morals from his work. Rather it was the practical conclusion of a physicist who sought to adopt quite pragmatically whatever approach would lead to successful physics.

We shall see that Einstein was indifferent and at times even derisive of considerations of mathematical simplicity early in his career. That was to change for good reason. We must understand Einstein quite literally when he proclaims in his manifesto (my emphasis): 'Our experience hitherto justifies us in believing that nature is the realisation of the simplest conceivable mathematical ideas'. This experience is not just the communal experience of scientists. It includes Einstein's own experiences; most importantly, it includes his experience with his greatest discovery, the general theory of relativity.

His indifference to mathematical simplicity persisted up to and through the years in which he worked on completing the theory. This indifference was weakened temporarily by the need to proceed within a context more mathematically sophisticated than any in which he had worked before. His research required him to master the Ricci Levi-Civita 'absolute differential calculus', now called 'tensor calculus'. As he formulated and tested candidate equations for his theory, he consciously alternated between two explicit strategies. The first employed physical constraints to narrow in on the final theory; the second asked after the laws that were most naturally written in the new formalism. He expected both to yield the same result so that one could be used to test the product of the other. When he erroneously concluded they did not yield the same results, he had to choose. He chose in favour of the first, the physical approach, unaware that an unnecessarily restrictive formulation of 
the physical constraints precluded the theory he would finally adopt at the end of $1915 .^{3}$

In 1913, the result was an ill-formed theory which Einstein struggled to embrace for over two years. He recognised his errors towards the end of 1915, just as the great mathematician David Hilbert turned his powers to the task of giving Einstein's theory an elegant axiomatic formulation. Einstein spent that November in mounting frenzy, sending a series of communications to the Prussian Academy of Science, each correcting the one before, all the while knowing that Hilbert was hard on his heels. The final equations were communicated by an exhausted Einstein at the end of the month. Through this last month, Einstein had consciously chosen to allow the natural mathematical constructions of the absolute differential calculus to guide him and that they did. They brought him rapidly to the final result, the most joyous achievement of Einstein's scientific life. The speed and ease of this final passage was an experience Einstein never forgot and, I will urge, were recalled by him when he needed to justify his new canon of mathematical simplicity. The canon grew more important in Einstein's thought and work and came to dominate his search for a unified field theory.

Section 2 below will review Einstein's early enthusiasm for mathematics and recount how Einstein consciously chose to neglect the use of more advanced mathematics in his physical theorising, right up to the moment at which tensor calculus was introduced into his search for the general theory of relativity. In Section 3, we shall see how Einstein, even at this last moment, was quite disparaging and even scornful of the heuristic power of mathematical simplicity. In Section 4 we begin to review how Einstein consciously alternated between answering the demands of physical constraints and natural mathematical conditions in devising the draft of his general theory of relativity, the 'Entwurf' theory. The next Section 5 reviews Einstein's return to the mathematically natural candidates for gravitational field equations and documents an immediate reversal in his appraisal of the importance of mathematical simplicity. The final Section 6 offers conclusions.

\section{Mathematics and the Young Einstein}

Contrary to popular myth, the young Einstein was not a poor student. He took a special interest in mathematics. It yielded to a conscious decision to minimise the mathematical sophistication of his earlier work in physics.

Many struggling students and their parents have found consolation in the stories that Einstein was himself a poor student and perhaps afflicted with some sort of learning disability. Comforting as these stories are, there is no

\footnotetext{
${ }^{3}$ This synoptic appraisal of Einstein's struggle was developed by an historical research group meeting in Berlin. See Section 4 below.
} 
evidence for them. Einstein was a good if not stellar student, regularly earning respectable grades. ${ }^{4}$ For our purposes, we learn from our most reliable sources that some of Einstein's earliest interests focused on mathematics and that he did very well at it. He recalled in his Autobiographical Notes (Einstein, 1949, pp. 8-9) the wonder he experienced at the age of 12 when he acquired a book on Euclidean geometry and that he was able, independently, to find a proof of Pythagoras' theorem. A short biography by his sister Maja (Stachel et al., 1987, pp. xlviii-lxvi) affirms the intensity of Einstein's engagement with mathematics at this time (ibid., p. 1xi): 'Play and playmates were forgotten. [ ... ] For days on end he sat alone, immersed in the search for solution, not giving up before he found it'. ${ }^{5}$ This fascination with geometry seems to antedate a similar, sustained interest in science. Around this time, a family friend, Max Talmud, introduced Einstein to a literature in science and philosophy. On Maja's report (ibid., p. lxii), we learn that ' $[\mathrm{h}]$ is scientific interests were broadened as a result; he was no longer solely engrossed in mathematics $[\ldots]$ '.

We recover a portrait of a young Einstein both able in and fascinated by mathematics. It is reinforced by his report that he taught himself calculus between the ages of 12 and 16 (Einstein, 1949, pp. 12-13) and by a note he wrote at the age of 17 (Stachel et al., 1987 Doc. 22) in which he recorded his plan to attend the polytechnical school in Zurich to study 'mathematics and physics'. Those who have studied Einstein's publications and private calculations in manuscript can have little doubt of Einstein's facility and freedom in whichever branch of mathematics he chose to employ.

So how are we to explain the repeated rumours of some sort of mathematical weakness on Einstein's part? They have a largely anecdotal basis, although presumably they reflect some self effacing remarks by Einstein. McCormmach (1976, pp. xxvii, xxix) reports two: the physicist F. A. Lindemann, having met Einstein at the 1911 Solvay conference related that Einstein 'says he knows very little mathematics'. McCormmach gives Smith (1962, p. 43) as the ultimate source. Again McCormmach reports: 'On his deathbed Einstein is said to have lamented to his son: "If only I had more mathematics!", with Michelmore (1962, p. 197) given as the source. ${ }^{6}$ Some of the most famous can be traced back to the great mathematician David Hilbert himself - or at least are attributed to him without proper citation. Philip Frank (1947, p. 206), Einstein's biographer and

\footnotetext{
${ }^{4}$ This is not always immediately apparent from the documentary evidence. For example, at the Argau Canton School in the year 1895-1896 he was awarded a grade of 1-2 in physics in each of the third and fourth quarters on a scale of 1 to 6 . But in the first quarter of 1896-1897, he was awarded a grade of 5-6 (Stachel et al., 1987, p. 16). As the editors of Volume 1 of the Einstein Papers reveal (p. 17, note 4), this variation betokened no inconsistency in Einstein's achievement. It merely reflected a change in the grading scheme. In 1895-1896, 1 was the highest grade and 6 the lowest; that was reversed in 1896-1897.

${ }^{5}$ Translations from Stachel et al. (1987) are from Beck (1987).

${ }^{6}$ Rudolf Jakob Hamm, as part of a longer description of his visit to Einstein in Berlin in 1917 when he was a 22 year old student, reported Einstein telling him ' $[\ldots]$ He maintains that he is a bad calculator $[\ldots]$ ' (quoted in Seelig, 1956, p. 155).
} 
successor at Prague, attributed two such double edged quips to Hilbert: 'Every boy in the streets of our mathematical Göttingen understands more about four-dimensional geometry than Einstein $[\ldots]$. Yet, despite that, Einstein did the work and not the mathematicians'. 'Do you know why Einstein said the most original and profound things about space and time that have been said in our generation? Because he learned nothing about all the philosophy and mathematics of time and space'. ${ }^{7}$

It seems to me that there is a natural explanation for the origin of these rumours. Compared to the average physicist and perhaps even mathematician, Einstein's mathematical powers were excellent. But Einstein did not compare himself to them. The natural comparison was with the mathematicians who took an interest in his theory. These included Hermann Minkowski, David Hilbert, Hermann Weyl and many more - a roster of mathematical brilliance that outshines all. Presumably the quips attributed to Hilbert above reflect similar standards. Compatible with this, when Einstein sought assistance, as he so often did, he turned to mathematicians who could aid him in the mathematical aspects of his work, recognising that his competence in mathematics did not match his singular insight in physics. ${ }^{8}$ His collaboration with the mathematician Marcel Grossmann on general relativity is best known of these. Einstein seems to have made a conscious decision early in his career not to pursue mathematical researches and this was then accompanied by a minimalist approach to the mathematical tools used in his early physical theories. ${ }^{9}$ He wrote in his Autobiographical Notes (1949, pp. 14-15) of his time as a student at the Zurich Polytechnic:

The fact that I neglected mathematics to a certain extent had its cause not merely in my stronger interest in the natural sciences than in mathematics but also in the following peculiar circumstance. I saw that mathematics was split up into numerous specialties, each of which could easily absorb the short lifetime granted to us. Consequently, I saw myself in the position of Buridan's ass, which was unable to decide upon any particular bundle of hay. Presumably this was because my intuition was not strong enough in the field of mathematics to differentiate clearly the fundamentally important, that which is really basic, from the rest of the more or less dispensable erudition. Also, my interest in the study of nature was no doubt stronger; and it was not clear to me as a young student that access to a more

\footnotetext{
${ }^{7}$ These quips can become even more distanced from their source. Hilbert's biographer, Constance Reid (1986, p. 142), repeats them in variant form without citation to any source.

${ }^{8}$ The mathematician, Ernst G. Straus, was Einstein's assistant in Princeton when Einstein wrote his Autobiographical Notes. Straus (1982, p. 422) related how Einstein often told him of his recognition as a student that he could not discern the important in mathematics unlike in physics, the story related in the passage quoted below, so 'that was the only reason I could be of any use to him'.

${ }^{9}$ At the same time as reporting Hilbert's quips, Frank (1947, p. 206) also attributes a corresponding suspicion of Göttingen mathematics to Einstein: 'The people in Göttingen sometimes strike me, not as if they wanted to help one formulate something clearly, but instead as if they wanted only to show us physicists how much brighter they are than we'.
} 
profound knowledge of the basic principles of physics depends on the most intricate mathematical methods. That dawned on me only gradually after years of independent scientific work.

Einstein's ensuing scientific work continued under the influence of this decision. His annus mirabilis of 1905 gave us his famous papers on Brownian motion, the light quantum and the special theory of relativity. These works employed little mathematics beyond the calculus of many variables.

That Einstein's work in special relativity could benefit from more imaginative mathematics was shown in 1907, famously, when Hermann Minkowski reformulated Einstein's theory as the geometry of a four-dimensional spacetime. Under the influence of the Göttingen mathematical establishment, this sophisticated geometrical approach to the theory flourished, in spite of Minkowski's early death in 1909. By 1911, the first text book on relativity (Laue, 1911) developed the theory in this more sophisticated mathematical garb as a matter of course. Einstein's publications continued to eschew this greater level of mathematical sophistication. The following year, Einstein continued his attempts to develop his insight of 1907 on how the relativity principle might be generalised to acceleration. The papers he published that year, essential intermediates in his passage to the general theory of relativity, use mathematics no more sophisticated than his work of 1905 and 1907. Thus Einstein reportedly joked that 'I myself can hardly understand Laue's book' (Frank, 1947, p. 206). That the remark could reflect a chosen distance from Minkowski's four-dimensional methods is suggested by the more somber remark to Valentin Bargmann that Einstein had found these methods 'superfluous learnedness' (Pais, 1982, p. 152). This attitude was about to change, but not before Einstein had put on record his disdain for the heuristic power of mathematical simplicity and beauty.

\section{Einstein Condemns Abraham's Theory of Gravitation in 1912}

In 1912, Einstein reveals his continued distrust of considerations of mathematical beauty and simplicity in an acrimonious dispute with Max Abraham. Einstein accuses him of allowing such considerations to engender an untenable theory of gravitation.

Upon his return to Zurich in August 1912, Einstein sought the mathematical assistance of his friend Marcel Grossmann. He learned from Grossmann that the instrument needed to carry his new theory of gravitation to completion was lying neglected in the mathematical literature. It was the absolute differential calculus of Ricci and Levi-Civita (1901). Using this instrument Einstein's efforts at generalising the principle of relativity through a gravitation theory reached a new level of mathematical sophistication and he was now close to a reversal of his opinion of the heuristic power of mathematics. We may wonder whether this reversal was not already underway by 1912. Einstein was seven years beyond the 
great discoveries of 1905 . He was no longer a mere patent clerk but was rising rapidly through a succession of desirable academic positions. At the same time, he had encountered two problems that taxed his powers: the problem of the quantum and the problem of gravity and general relativity. Might these pressures have already caused him to rethink his brash, youthful appraisal of mathematics?

While we cannot preclude such a reappraisal in the deeper recesses of his heart, his remarks to his correspondents give no evidence of it prior to his exposure through Grossmann to the absolute differential calculus. Indeed they suggest his attitude hardened. Remarks in his letters on Abraham's new theory of gravitation suggest that Einstein thought reliance on such considerations a worthy target of public criticism and that Abraham had been induced by them to advance a defective theory. If Einstein was later to learn from his experience with general relativity of the value of a mathematical heuristic, then Einstein might well be learning from Abraham's theory of the power of the mathematical heuristic to lead one astray.

When Abraham's theory first appeared in January 1912, Einstein was quite taken by it exactly because of its mathematical appeal. He recalled in a letter to Besso of 26 March 1912: ' [ ... ] at the first moment (for 14 days!) I too was totally "bluffed" by the beauty and simplicity of his formulas'. ${ }^{10}$ By the end of the month, however, he had turned against the theory and, in the months following made sure to inform correspondent after correspondent that the theory was:

'totally untenable' (to Paul Ehrenfest, 12 February 1912 (Klein et al., 1993, Doc. 357)), 'incorrect in every respect' (to H. A. Lorentz, 18 February 1912 (Klein et al., 1993, Doc. 360)), 'totally unacceptable' (to Wilhelm Wien, 24 February 1912 (Klein et al., 1993, Doc. 365)), 'totally untenable' (to Heinrich Zangger, received 29 February 1912 (Klein et al., 1993, Doc. 366)), and

'completely untenable' (to Michele Besso, 26 March 1912 (Klein et al., 1993, Doc. 377)).

As the months passed and the dispute with Abraham deepened, his negative appraisals became more figurative. By 16 August 1912, he wrote to Ludwig Hopf (Klein et al., 1993, Doc. 416): ${ }^{11}$

Recently in two massive attacks - as you perhaps saw-Abraham slaughtered me, along with the theory of relativity, and wrote down (Phys. Zeitschr.) the only correct theory of gravitation (with the 'nostrification' of my results) - a stately beast, but it lacks three legs!

\footnotetext{
${ }^{10}$ Klein et al., 1993, Doc. 377. Translations from this volume here and below are based on Beck (1995) with my adjustments.

${ }^{11}$ Again on 29 October 1912, he wrote to Arnold Sommerfeld (Klein et al., 1993, Doc. 421): ‘[ ... ] Abraham's new theory is, as far as I can see, logically correct, it is nevertheless a monster spawned by embarrassment'.
} 
The initial aesthetic appeal of Abraham's theory arose because it supplied the simplest accommodation of Newton's theory of gravitation to Minkowski's four-dimensional spacetime (see Appendix A: 'Abraham's Theory of Gravitation'). But Einstein soon saw past it. To avoid the physical triviality of dealing only with constant potential fields, Abraham had chosen to copy Einstein's idea of allowing the speed of light $c$ to vary with the gravitational potential. Einstein soon realised that, in Abraham's hands, this modification compromised the essential structure of Minkowski spacetime. ${ }^{12}$ Einstein began to press Abraham both in private correspondence and in publication, showing that with variable $c$ the Lorentz transformations would no longer hold, even infinitesimally. Abraham incorporated Einstein's criticism into the development of his theory but also began to query the validity of the (special) theory of relativity and whether Einstein's own gravitation theory, which employed a variable $c$, had given up this theory as well. (For a compact synopsis of the dispute, see Klein et al., 1995, pp. 124-127.) As the dispute with the notoriously acidic Abraham grew unseemly, Einstein decided to break away from further discussion with a short note in Annalen der Physik (Einstein, 1912b), addressed not to Abraham but the journal's readers. He explained that he would not reply further to Abraham and asked readers not to understand his silence as agreement between Einstein and Abraham.

Einstein made clear to his correspondents that Abraham had let himself be led astray by mathematical considerations just as Einstein himself had initially been 'bluffed'. He wrote to Heinrich Zangger on 27 January 1912 (Klein et al., 1993, Doc. 344):

Abraham has extended my gravitation item to a closed theory, but he made some serious mistakes in reasoning so that the thing is probably wrong. This is what happens when one operates formally, without thinking physically!

Again he wrote to Besso on 26 March 1912 (Klein et al., 1993, Doc. 377):

Abraham's theory has been created out of thin air, i.e. out of nothing but considerations of mathematical beauty, and is completely untenable. How this intelligent man could let himself be carried away with such superficiality is beyond me. To be sure, at the first moment (for 14 days!) I too was totally 'bluffed' by the beauty and simplicity of his formulas.

Einstein even suggested that such formal thinking was a lazy and ineffective way of doing physics. He asked Wien (24 February 1912, Klein et al., 1993, Doc. 365): 'Who has ever been so lucky to hit upon correct equations without any effort!' We may well wonder if Einstein carried these warnings against purely formal thinking and the over-valuing of mathematical simplicity and beauty into

\footnotetext{
${ }^{12}$ He wrote to Wilhelm Wien on 11 March 1912 (Klein et al., 1993, Doc. 371): '[ ... ] the thing is not as simple as Abraham thinks. In particular, the principle of the constancy of $c$ and, with it, the equivalence of the 4 dimensions are lost'.
} 
the intense work on the general theory of relativity that he was about to undertake.

\title{
4. Einstein's Struggles to Find the Gravitational Field Equations of General Relativity
}

\begin{abstract}
We learn from an historical research group in Berlin that Einstein consciously balanced formal and physical thinking in these struggles. He allowed erroneous physical restrictions to dominate the formal and published formally deficient equations. His completion of the theory was delayed for over two years.
\end{abstract}

With his return to Zurich in August 1912 and his immersion in the absolute differential calculus of Ricci and Levi-Civita, Einstein's antipathy to mathematical indulgences almost immediately began to soften. He wrote to Arnold Sommerfeld on 29 October 1912 (Klein et al., 1993, Doc. 421): ${ }^{13}$

I am now working exclusively on the gravitation problem and believe that I can overcome all difficulties with the help of a mathematician friend of mine here [Marcel Grossmann]. But one thing is certain: never before in my life have I toiled any where near as much, and I have gained enormous respect for mathematics, whose more subtle parts I considered until now, in my ignorance, as pure luxury. Compared with this problem, the original theory of relativity is child's play.

When he wrote these words, Einstein had over two years of toil ahead before his theory would be completed in November 1915.

By mid 1913, Einstein had arrived at a first draft of his new gravitation theory, which he published in collaboration with Grossmann, the 'Entwurf' theory (Einstein and Grossmann, 1913). That theory contained virtually all the elements of the final general theory of relativity. It associated gravitation with a curvature of spacetime and used the Ricci Levi-Civita calculus to ensure that all its equations were generally covariant, that is, remained unchanged in form under arbitrary transformation of the spacetime coordinate system. The one exception was its gravitational field equations. These were the most important equations of the theory, specifying how matter curves spacetime and how that curvature propagates. These equations were not generally covariant. That contradicted almost everything associated with the theory, including Einstein's initial demand that they be so, the framework of the Ricci Levi-Civita calculus, the final version of the theory and the expectations of any modern reader who happens to stumble onto Einstein and Grossmann's paper.

\footnotetext{
${ }^{13}$ Presumably Einstein wrote of his reappraisal of mathematics with the understanding that it would be read by welcoming eyes. Sommerfeld had long sought to propagate Minkowski's new mathematical methods. By this time he had authored an introductory survey paper on four-dimensional methods in Annalen der Physik (Sommerfeld, 1910). He also wrote detailed explanatory notes on Minkowski's (1908) famous popular lecture 'Space and Time' and they are included in the standard edition cited here.
} 
A research group, meeting in Berlin over the last few years, ${ }^{14}$ has come to a most enlightening understanding of Einstein's efforts to construct and complete his general theory of relativity. ${ }^{15}$ Einstein sought to construct a theory that satisfied a list of requirements. The theory must revert to Newtonian theory in the case of sufficiently weak gravitational fields. It must satisfy a requirement of conservation of energy and momentum. It must embody the principle of equivalence, which demands that we generate a homogeneous gravitational field in a Minkowski spacetime by transforming to a uniformly accelerating frame. Finally it must embody a generalisation of the principle of relativity that includes acceleration. Part of the latter was an expectation that it would account for the inertia of bodies as arising from an interaction with all the other masses of the universe. This requirement was later called 'Mach's Principle' and separated from the generalised principle of relativity.

Einstein divided these requirements and their consequences loosely into two groups. The first had a physical character and included the requirement of the Newtonian limit and of conservation of energy and momentum. The second group had a more formal, mathematical character. Both the principle of equivalence and the generalised principle of relativity were expressed mathematically by Einstein as a requirement of the covariance of the equations of his theory. ${ }^{16}$ To implement them, these equations should remain unchanged in form under coordinate transformations that corresponded to transformations to accelerated frames of reference. While narrower covariance might be sufficient, Einstein found it most convenient to require general covariance; that is, the equations must remain unchanged in form under arbitrary transformation of the coordinates. ${ }^{17}$ This was his principle of general covariance.

${ }^{14}$ The group's members include Michel Janssen, John Norton, Jürgen Renn, Tilman Sauer and John Stachel. The research group was begun under the direction of Peter Damerow and Jürgen Renn with the Working Group Albert Einstein, funded by the Senate of Berlin and affiliated with the Center for Development and Socialization headed by Wolfgang Edelstein at the Max Planck Institute for Human Development and Education in Berlin. It was continued under the direction of Jürgen Renn as part of the project of studies of the integration and disintegration of knowledge in modern science at the Max Planck Institute for the History of Science.

${ }^{15}$ A preliminary survey of this account of Einstein's heuristics has been presented by Renn and Sauer (1999) and a more extensive and detailed volume is now in preparation. For a related proposal on how Einstein arrived at general relativity by restricting a universe of candidate theories with diverse heuristic principles, see Norton (1995). See this paper also for briefer remarks on how Einstein's experience with general relativity altered his perception of the heuristic value of mathematics.

${ }^{16}$ For an account of how Einstein translated the principle of equivalence into a covariance requirement, see Norton (1985). Whether Einstein's translation of relativity principles into covariance principle is legitimate remains a subject of often heated debate. For a review, see Norton (1993).

${ }^{17}$ Just which were the 'arbitrary' transformation was left vague by Einstein. They certainly included all $\mathrm{C}^{\infty}$ transformations. General covariance requires more than a generalised principle of relativity since it would include covariance under transformations that do not correspond to changes of states of motion, such as the replacement of Cartesian spatial coordinates by polar coordinates. 
Each group of requirements exerted powerful constraints on the admissible theories. Merely asking how one group could be satisfied in a simple and natural way would greatly reduce the choices. This fact induced Einstein to employ a two-pronged strategy. He might start with the physical requirements, for example, and construct components of the theory naturally suggested by them. He could then test them against the formal, mathematical requirements. That is, he would ask if they have the requisite covariance. If all is well they will and the two sets of requirements are satisfied. Conversely, he might look in the mathematics literature, with the assistance of Marcel Grossmann, for generally covariant expressions that appear suitable for his theory. He could then check whether they satisfy the physical requirements.

The Berlin group arrived at this account of Einstein's methods in part from the examination of a notebook of calculations Einstein kept during the period of his work on the 'Entwurf' theory, the 'Zurich Notebook'. ${ }^{18}$ This remarkable notebook documents Einstein's deliberations from his early acquaintance with the new methods of Ricci and Levi-Civita, through his evaluation of candidate gravitational field equations, to the final equations of the 'Entwurf' theory.

Early in these deliberations we find a simple example of the successful application of the strategy (see Appendix B: 'Einstein Uses Physical and Formal Mathematical Approaches to Write the Energy Conservation Law'). Einstein sought the expression for the conservation of energy and momentum in the case of distributed matter, such as a fluid flowing under the influence of a gravitational field. He began with the equation of motion of a single point mass in free fall. From it he read off expressions for gravitational force and for the energy and momentum of the mass. Conceiving the fluid as a cloud of masses, he converted these expressions to densities and assembled a simple differential law that set the gravitational force density equal to the rate of accumulation of energy-momentum density and arrived at his final law.

This result derived essentially from physical reasoning. While the result was strongly suggested by Einstein's analysis, its derivation was not an unequivocal translation of the equation of motion of individual masses into the corresponding law for distributed matter. Einstein needed a way to check the result. That was provided by his formal, mathematical requirements. In the best case, the law he found would not just be generally covariant. It would be one that could be assembled from simple elements within the Ricci Levi-Civita framework. To see whether this was so, Einstein broke the equation into two parts, a differential operator and the stress-energy tensor it acted on. The general covariance of the equation was assured if that differential operator had quite general powers, so that it would generate generally covariant expressions when applied not just to the stress-energy tensor, but to any tensor like it. To test this, Einstein let the operator act on the other, similar tensor conveniently at hand, the metric tensor, and confirmed the general covariance of the resulting equation. Einstein's

\footnotetext{
${ }^{18}$ So named because its content was written in Zurich. See Klein et al. (1995, Doc. 10). The Berlin group will also publish a more extensive examination of this notebook in their joint work in preparation.
} 
expectations were fulfilled. There were two paths to the result. The one he followed used essentially physical reasoning. But he could also have arrived at the same result by seeking simple expressions of the appropriate form within the Ricci Levi-Civita calculus. Einstein's obvious satisfaction with the outcome is recorded by a single word written at the bottom of the page: 'Stimmt' ['correct'].

While this simple application of his strategy succeeded, Einstein was not so fortunate when he turned his efforts to the gravitational field equations. There his attempts to apply this same strategy met with repeated failure, in spite of proposals of increasing ingenuity and ever greater exertions. On page after page of the notebook he approached the problem from either side, here writing expressions suggested by the physical requirements of the Newtonian limit and energy-momentum conservation, there writing expressions naturally suggested by the generally covariant quantities supplied by the mathematics of Ricci and Levi-Civita. Try as he did, he could not get the two groups of requirements to agree. The expressions generated by physical requirements either lacked sufficient covariance or had unknown covariance. Conversely, the expressions generated by covariance considerations failed to yield the correct Newtonian limit or energy-momentum conservation. Most famously, Einstein considered gravitational field equations based on the Riemann-Christoffel curvature tensor, now the natural and rapid entry to the modern gravitational field equations. Grossmann reported their failure to succeed with this tensor in his part of Einstein and Grossmann (1913, Part II, p. 36). ${ }^{19}$

As Einstein's deliberations drew to a close, he was forced to a most unwelcome decision. He could not be assured of satisfaction of both requirements. $\mathrm{He}$ could offer equations that demonstrably satisfied only one group. Which would it be? Einstein had to choose and his decision would haunt him for over two years. He chose gravitational field equations that were assured to satisfy energy-momentum conservation and return the correct Newtonian limit. But their covariance was unknown. These were the equations he published in Einstein and Grossmann (1913), the 'Entwurf' paper.

Einstein later discovered that his dilemma was of his own making. There are equations that satisfy all his conditions. But he had erroneously judged them as inadmissible by formulating some of his requirements too restrictively. Most importantly, he presumed that the geometry of space must become flat in the weak fields that return Newtonian theory and would not allow small deviations from flatness in quantities of the order of smallness. ${ }^{20}$

\footnotetext{
${ }^{19}$ The saga of Einstein's heroic struggle has been of considerable interest to historians of general relativity and an extensive literature has grown around it. For my contribution, see Norton (1984). See also the commentary in Klein et al. (1995, Doc. 10).

${ }^{20}$ While these presumptions about the Newtonian limit were sufficient to preclude Einstein's acceptance of his final theory of November 1915, Einstein clearly also allowed one or more other misconceptions to derail his investigations. Just what they were remains under debate. My own proposal is that it was the same error committed months later in the context of the 'hole argument'. Translated back to the context of the Zurich notebook, it led Einstein to place additional, impossible demands on how the gravitational field equations must yield the Newtonian limit. See Norton (manuscript a, manuscript b).
} 
These insights would not come until much later in 1915. In the meantime Einstein laboured on, trying to convince himself of the admissibility of his defective theory and that the natural mathematical expressions were merely deceptive temptations. In early March 1914, after he sensed some success, he reported to his friend Besso how these efforts had worn him (Klein et al., 1993, Doc. 514):

At the moment I do not especially feel like working, for I had to struggle horribly to discover what I described above. The general theory of invariants was only an impediment. The direct route proved to be the only feasible one. It is just difficult to understand why I had to grope around for so long before I found what was so near at hand.

These remarks are also revealing for they affirm that Einstein consciously conceived his efforts in terms of the two routes to the final result. ${ }^{21}$ The 'direct route', his usual physical argumentation, turned out to work. The formal, mathematical approach would look to the theory of invariants to suggest simple covariant expressions that would be candidates for the theory's laws. These expressions proved to be inadmissible physically. While he had been tempted, he now reverted to his earlier skepticism about more advanced mathematics. For physicists it is a distracting luxury after all.

\section{Reversal at the Eleventh Hour}

In the fall of 1915, aware of the flaws in his 'Entwurf' theory, Einstein decided he could only find the correct theory through the expressions naturally suggested by the mathematics. He proceeded rapidly to the completion of the theory and the greatest triumph of his life, barely outpacing Hilbert and just securing his priority. Einstein now saw the magic in mathematics.

Einstein remained reconciled to his flawed theory through his move to Berlin later in April 1914, through the writing of an expansive review article (Einstein, 1914; submitted 29 October) and on into the summer of the following year. In retrospect, Einstein's efforts to sustain this reconciliation were contrived and ill-fated. If he could not demonstrate the general covariance of his theory, he would do the next best thing. He would try to show with his 'hole argument' that general covariance was not to be desired after all; it would be physically uninteresting since, he urged, it would violate the determinism of the theory (Einstein, 1914, Section 12). And then he thought he could derive his 'Entwurf' gravitational field equations largely from the demand that they have the maximum covariance permitted by the hole argument (Einstein, 1914, Section 15).

\footnotetext{
${ }^{21}$ Were these categories already in Einstein's thought before he commenced working in the Ricci Levi-Civita calculus? That they were is suggested by his remark of January 1912 to Zangger (quoted above): ' $[\ldots]$ This is what happens when one operates formally, without thinking physically!'
} 
The fragile truce could not last. Einstein had long known that his new theory of gravitation did not account for the anomalous motion of Mercury's perihelion (see Klein et al., 1995, Doc. 14). That could not yet be counted as so serious a defect since no other theory could then do better. His confidence would soon crumble. On 30 September 1915, a shaken Einstein sent disturbing news to the astronomer Erwin Freundlich (Schulmann et al., 1998, Part A, Doc. 123). Contrary to his earlier belief, he now found his 'Entwurf' theory was not covariant under a simple transformation that set the spatial coordinate axes into uniform rotation. This discovery was shocking since it meant that his theory could not extend the relativity of motion to such important cases as Newton's rotating bucket of water. ${ }^{22}$ In obvious despair, he confided to Freundlich and vainly sought help in solving the problem that had exercised and defeated Einstein at the height of his powers: ${ }^{23}$

This is a blatant contradiction. I do not doubt, therefore, that the theory of the perihelion motion also suffers from the same mistake. Either the equations are already numerically incorrect (number coefficients) or I apply the equations in a fundamentally wrong way. I do not believe I am able to find the mistake myself, for in this matter my mind is too set in a deep rut. More likely, I have to rely on some fellow human being with unspoiled brain matter finding the mistake. Do not forget to study the subject, if you have time [Einstein's emphasis].

This discovery broke Einstein's confidence in his 'Entwurf' theory and he soon recognised further deficiencies in it. ${ }^{24}$ By November, Einstein had pressed his spoiled brain matter into the most productive service. He sent a series of communications to the Prussian Academy (Einstein, 1915a,b,c,d) in which he wound his way out of the labyrinthine confusions of the 'Entwurf' theory, demonstrated the successful explanation of the anomalous perihelion motion of Mercury and, in stages, offered the generally covariant gravitational field equations that now bear his name.

The feat brought Einstein the greatest success of his life, the satisfactory completion of the general theory of relativity. He wrote to Sommerfeld at the end of November (Schulmann, 1998, Part A, Doc. 153, 28 November 1915; Einstein's emphasis):

You must not be mad with me that I only now reply to your friendly and interesting letter. But in the last month I had one of the most exciting and strenuous times of my life, also though one of the most successful. I could not think about writing.

Its moment of greatest triumph came when he found that his theory now explained the anomalous motion of Mercury's perihelion exactly without the

\footnotetext{
${ }^{22}$ For an account of Einstein's joust with rotation, see Janssen (1999).

${ }^{23}$ Translation based on Janssen (1999, p. 135).

${ }^{24}$ For further discussion of Einstein's return to general covariance, see Norton (1984, Section 7; manuscript a, Section 11).
} 
need for any arbitrary assumptions. That result gave him special pleasure as he recounted the following January in a letter to Ehrenfest (Schulmann, 1998, Part A, Doc. 182): ${ }^{25}$

Imagine my joy at the recognition of the feasibility of general covariance and at the result that the equations correctly yield the perihelion motion of Mercury. I was beside myself for several days in joyous excitement.

In a similar exuberant humour, he wrote to Besso (Schulmann, 1998, Part A, Doc. 162, 10 December 1915; Einstein's emphasis):

My wildest dreams have been fulfilled. General covariance. Perihelion motion of Mercury wonderfully exact.

We can gauge how excited he must have been by the fact that this successful explanation remains today one of the favourite illustrations in philosophy of science of striking success in confirmation.

How had Einstein hauled his spoiled brain matter out of its rut? To what did he owe his triumph and these moments of greatest joy? He gave his answer in print in the opening pages of Einstein (1915a, p. 778), the first paper of that memorable November series: ${ }^{26}$

[...] I completely lost trust in the field equations I had chosen and looked for a way to restrict the possibilities in a natural manner. Thus I went back to the requirement of a more general covariance of the field equations, which I had left only with a heavy heart when I worked together with my friend Grossmann. In fact we had then already come quite close to the solution of the problem given in the following.

${ }^{25}$ In judging his success with Mercury the strongest experience emotionally in Einstein's scientific life, Pais (1982, p. 253) reports even more powerful remarks by Einstein over the impact on him of this result:

The discovery was, I believe, by far the strongest emotional experience in Einstein's scientific life, perhaps in all his life. Nature had spoken to him. He had to be right. 'For a few days, I was beside myself with joyous excitement.' $[\ldots]$ Later, he told Fokker that his discovery had given him palpitations of the heart $[\ldots]$. What he told de Haas is even more profoundly significant: when he saw that his calculations agreed with the unexplained astronomical observations, he had the feeling something actually snapped in him...

(The last ellipses are Pais'.) For further details of Einstein's calculation see Earman and Janssen (1993).

${ }^{26}$ In this first communication, Einstein had not yet arrived at his final equations. (For further discussion of how the proposals developed through the four communications of November 1915, see Norton, 1984, Section 8.) In the first communication, he advanced gravitational field equations that were not generally covariant, but only covariant under the broad class of transformations of unit determinant. Hence he speaks of 'more general covariance' and not simply 'general covariance'. The field equations he is about to advance had been considered in the Zurich Notebook but rejected for reasons that have been obscure until recently. Norton (manuscript a, b) reports a conjecture that I believe explains naturally why the proposal was abandoned in the Zurich Notebook. 
He gave a similar account in correspondence to Sommerfeld at the end of the month (Schulmann, 1998, Part A, Doc. 153):

Once all trust in the results and methods of the earlier theory had gone, I saw clearly that a satisfactory solution could only be found through a connection to the general theory of covariants, i.e. to Riemann's covariant.

In effect his new tactic was to reverse his decision of 1913 . When the physical requirements appeared to contradict the formal mathematical requirements, he had then chosen in favour of the former. He now chose the latter and, writing down the mathematically natural equations, found himself rapidly propelled towards a theory that satisfied all the requirements and fulfilled his 'wildest dreams'.

There was a heightened sense of urgency and a sinister cast in this last all-consuming month. The previous summer, Einstein had visited Göttingen to speak on his earlier 'Entwurf' theory. He had found to his delight that the mathematicians there, notably the great Felix Klein and David Hilbert, understood and accepted his theory. ${ }^{27}$ Hilbert had now also turned to work on the theory and, in the course of that final November, was also on the trail of the gravitational field equations. Einstein might have apologised to Sommerfeld for being too distracted to write to him in that month. But he certainly found time to correspond with Hilbert and each tried nervously to discern the extent of agreement with the equations the other was advancing. Einstein presented his final equations on 26 November 1915, to the Prussian Academy. Hilbert's (1915) communication of a paper with the same equations to the Göttingen Academy is dated 20 November 1915.

Hilbert was not quite as far ahead as a simple comparison of these two dates would suggest. This was shown by the recent discovery in the Göttingen archive of the proof pages of Hilbert's (1915) paper (Corry et al., 1997). These proof pages were revised substantially to yield the final published paper. In the unrevised version, the equations in a form comparable to those communicated by Einstein on 26 November are not in evidence. Nonetheless, Hilbert was extremely close. ${ }^{28}$ The unrevised proof pages do contain exactly the recipe for Einstein's equations: they are to be recovered by varying an action density that is Riemann's curvature scalar. Carrying through the recipe had an assured result and required no special mathematical brilliance.

Einstein would be fully justified in November in fearing that he might lose the consummation of his theory to Hilbert. While history now grants Einstein the prize, the immediate effect of his experience was a temporary bitterness concerning Hilbert. He wrote to Zangger on the day of communication of the final result (Schulmann et al., 1998, Part A, Doc. 152, 26 November 1915; Einstein's

\footnotetext{
${ }^{27}$ See Howard and Norton (1993) for an account of this visit and a proposal concerning an unexpected repercussion.

${ }^{28}$ The quite stunning revelation from the unrevised proof pages is that Hilbert there accepts Einstein's hole argument against general covariance.
} 
emphasis):

The theory is of incomparable beauty. However only one colleague has really understood it and that one seeks to 'nostrify' it in a clever manner (Abraham's expression). I have in my personal experience hardly ever gotten to know better the contemptibility of humanity as on the occasion of this theory and everything associated with it. But it does not bother me.

Einstein soon made his peace with Hilbert. ${ }^{29}$

Einstein's reversal was his Moses that parted the waters and led him from bondage into the promised land of his general theory of relativity - and not a moment too soon. Had he delayed, the promised land might well have been Hilbert's. Einstein (1933b, p. 289) recalled how he 'ruefully returned to the Riemann curvature'. He now saw just how directly the mathematical route had delivered the correct equations in 1913 and, by contrast, how treacherous was his passage if he used physical requirements as his principal compass. While still sparring with Hilbert in the middle of that frantic November, he described to Hilbert (Schulmann et al., 1998, Part A, Doc. 148, 18 November 1915) wistfully how the mathematical pathway had actually already given him the right equations three years before:

The difficulty did not lie in finding generally covariant equations for the $g_{\mu v}$; this is easily done with the help of Riemann's tensor. Rather it was difficult to recognise that these equations formed a generalisation of Newton's law and indeed a simple and natural generalisation. I succeeded in this only in the last few weeks $[\ldots]$, while I had already taken into consideration the only possible generally covariant equations, which now prove to be the right ones, three years ago with my friend Grossmann. Only with heavy hearts did we detach ourselves from them, since the physical discussion had apparently shown their incompatibility with Newton's law.

Within weeks, Einstein was quite happy to pronounce bluntly that the equations suggested by the mathematics were the obvious ones, but that physical considerations had distracted him. ${ }^{30}$ He wrote to Besso on 10 December 1915 (Schulmann et al., 1998, Part A, Doc. 162):

This time the most obvious was correct; however Grossmann and I believed that the conservation laws would not be satisfied and that Newton's law would not come out in the first approximation.

The reversal was complete. Just a few years before, Einstein had growled in derision over thoughts of mathematical beauty and simplicity. He now found

\footnotetext{
${ }^{29}$ Einstein and Hilbert's interactions over general relativity have long been a subject of historical investigation. See Earman and Glymour (1978), Mehra (1974), Norton (1984, Section 8), Pais (1982, pp. 257-261). They have been enhanced greatly by recent work. See Corry (1999), Renn and Stachel (1999) and Sauer (1999).

${ }^{30}$ I am grateful to Michel Janssen for pointing out the irony of these remarks. Less than two years before, Einstein had told this same Besso just the opposite: the physical argumentation gave the obvious result; the mathematics was a distraction! (See above.)
} 
that mathematics set his teenage heart aflutter. He wrote in the introduction to the first of his communications of 4 November 1915 (1915a, p. 779):

Hardly anyone who has truly understood it can resist the charm of this theory; it signifies a real triumph of the method of the general differential calculus, founded by Gauss, Riemann, Christoffel, Ricci and Levi-Civita.

Einstein had succumbed to these charms before he knew that his theory was about to deliver the triumph of explaining Mercury's anomalous motion-that he found later in the month. ${ }^{31}$

Einstein's reversal was heartfelt and embraced with all the relief of the survivor of an unforeseen peril. But how close had it brought him to the viewpoint of the grand manifesto of the Herbert Spenser lecture? In his November 1915 remarks, he praised the advent of general relativity as a triumph of the 'method' of the general differential calculus. That suggests that he had already seen mathematics as hosting what he called 'the creative principle' and the 'right way' in his 1933 Herbert Spenser lecture. If that was his view, however, why did it take nearly two decades before Einstein gave the view canonical public formulation? Was it just that he saw no need to do it earlier? Or was it that the ideas were so radical that he was reluctant to proclaim them universally in all their shocking details? Or had he himself not realised how radical the viewpoint was that he was drawn towards? His experiences of November 1915 might have been life changing, but might he have needed over a decade to assimilate their full significance consciously and find words to give it concrete form? Or might he have been reluctant and needed reinforcement from further experiences before he was driven to the viewpoint of 1933 ?

How might we answer these questions? I am grateful to Michel Janssen for the astute suggestion that we look at Einstein's 1918 response to Hermann Weyl's (1918) proposal for a geometrical unification of gravitation and electromagnetism, for there we find Einstein again balancing mathematical and physical considerations. ${ }^{32}$ Weyl had found a mathematically ingenious way of extending the Riemannian geometry of general relativity so that it would accommodate electromagnetism as well as gravitation. He introduced a second group of transformations of the field quantities that soon came to be known as 'gauge transformations'. The proposal faltered and languished, no doubt in large measure because of Einstein's objections and opposition, but the mathematical device of the gauge transformation became wildly successful in a different context and now drives the flourishing tradition of gauge theories in quantum field theory.

\footnotetext{
${ }^{31}$ That his experience with the completion of the theory was life changing is suggested by power of his concluding remarks in a lecture outlining how he discovered the theory (1933b, pp. 289-290): 'In the light of the knowledge attained, the happy achievement seems almost a matter of course, and any intelligent student can grasp it without too much trouble. But the years of anxious searching in the dark, with their intense longing, their alternations of confidence and exhaustion and the final emergence into the light—only those who have experienced it can understand that'.

${ }^{32}$ For further discussion, see Schulmann et al. (1998, Part A, pp. lii-liii). Einstein communicated Weyl's paper to the Prussian Academy with his own note of reservation, Einstein (1918b).
} 
Einstein's response to the proposal was rapid and immovable. He marvelled at its mathematical brilliance but insisted that a simple physical objection was fatal to it. Weyl's theory allowed the magnitude of measuring rods and clock ticks to vary with the paths the rods and clocks followed in spacetime. Atomic emissions spectra are the ticking of a kind of atomic clock. So, Einstein concluded, sharp atomic spectral lines would not be possible in Weyl's theory, since the frequency of an atomic clock would depend on its history, contrary to our experience. As soon as Einstein received Weyl's paper he formulated his double-edged response (Einstein to Weyl, 6 April 1918; Schulmann et al., 1998, Doc. 498. Translations here and below from Hentschel (1998). Einstein's emphasis):

Your paper has arrived. It is a first class stroke of genius. However I have not been able to settle my measuring-rod objection yet. More details on this another time.

Einstein persisted in his mixture of praise of the mathematics and rejection of the physics two days later (Einstein to Weyl, 8 April 1918; Schulmann et al., 1998, Part B, Doc. 499):

Your chain of reasoning is so wonderfully self-contained. The deduction of the dimension number 4 impressed me very much as well. The decomposition of your invariant of 'weight' zero is also very striking. Except for the agreement with reality, it is in any case a grand intellectual achievement.

How are we to understand Einstein's response to Weyl's theory? Suggestive as his remarks are, I do not see how they allow us to decide how close Einstein had come in 1918 to the views expressed in 1933. He praised Weyl's elegant mathematics but unhesitatingly rejected the physical theory that resulted. Might this reveal that Einstein had not yet fully succumbed to the charms of mathematics? Perhaps the radical viewpoint of 1933 had not yet fully taken hold but was still advancing quietly on Einstein's consciousness. Or this combined praise might well just be reminding us of an element present in the 1933 viewpoint all along. While the Einstein of 1933 proclaimed that 'the creative principle resides in mathematics', he was equally clear that this creative principle was not free of restriction: 'Experience remains, of course, the sole criterion of the physical utility of a mathematical construction'. While the creative principle flourished sumptuously in Weyl's work of 1918, its product failed to meet experience, the 'sole criterion of [its] physical utility'. The Einstein of 1918 would be well justified in dismissing Weyl's invention as a viable physical proposal, no matter how close he had come to the viewpoint of 1933.

\section{Conclusion}

A passage in Einstein's Autobiographical Notes recalls how his experience with gravitation taught him the heuristic power of mathematics. 
By the time of his 1933 manifesto, Einstein had completely discarded his youthful skepticism over the heuristic power of mathematics and forcefully advocated the exact opposite view. No doubt many factors contributed to his change of heart. Of these factors, his experience with general relativity must count as the most important. Indeed this seems to be just the message he sent when he reflected in his Autobiographical Notes (Einstein, 1949, p. 85) on the difficulty of finding the fearfully complicated equations that govern his unified field theory. He wrote:

I have learned something else from the theory of gravitation: no collection of empirical facts however comprehensive can ever lead to the setting up of such complicated equations [as non-linear field equations of the unified field]. A theory can be tested by experience, but there is no way from experience to the construction of a theory. Equations of such complexity as are the equations of the gravitational field can be found only through the discovery of a logically simple mathematical condition that determines the equations completely or almost completely. Once one has obtained those sufficiently strong formal conditions, one requires only little knowledge of facts for the construction of the theory; in the case of the equations of gravitation it is the four-dimensionality and the symmetric tensor as expression for the structure of space that, together with the invariance with respect to the continuous transformation group, determine the equations all but completely.

When he wrote here of learning from the theory of gravitation - general relativity - we cannot doubt what Einstein had in mind. It was his three errant years of struggle to complete the theory; his last minute reversal over the heuristic power of mathematics; how it had allowed him to bring the theory to a rapid, triumphant close; and how he won his race with Hilbert. In retrospect, Einstein could see that it was so straightforward. He merely needed to let simple mathematical conditions dictate his equations; he then would have to bother little with physical facts. In 1913, when working on the general theory of relativity, he had not approached the problem this way. He had now learned his lesson. He would not make the same mistake with his unified field theory.

\section{Appendix A. Abraham's Theory of Gravitation}

It is the simplest four-dimensional extension of Newton's theory. It runs into difficulties that Abraham chose to resolve by allowing $c$, the speed of light, to vary with the gravitational potential. Einstein was very critical of this escape.

Abraham's (1912) theory was initially intended to be a simple and natural accommodation of Newton's theory of gravitation to relativity theory, that is, it would 'satisfy the principle of relativity' $(1912$, p. 1). Its leading idea was to take the equations of Newton's theory written in three-dimensional space and generalise them directly to a four-dimensional spacetime. In Newton's theory, the force $\left(F_{x}, F_{y}, F_{z}\right)$ on a unit mass was merely the gradient of gravitational 
potential $\Phi$ and the potential was in turn fixed by the mass density $v$ according to Poisson's equation

$$
F_{x}=-\frac{\partial \Phi}{\partial x}, \quad F_{y}=-\frac{\partial \Phi}{\partial y}, \quad F_{z}=-\frac{\partial \Phi}{\partial z}, \quad \frac{\partial^{2} \Phi}{\partial x^{2}}+\frac{\partial^{2} \Phi}{\partial y^{2}}+\frac{\partial^{2} \Phi}{\partial z^{2}}=4 \pi \gamma \nu,
$$

where $x, y$ and $z$ are Cartesian spatial coordinates and $\gamma$ is the gravitation constant. The relativised version was generated merely by adding an extra coordinate $u=i c t$ to extend the space into a spacetime then extending the above formulae with terms exactly mimicking those of Newton's theory,

$$
\begin{gathered}
F_{x}=-\frac{\partial \Phi}{\partial x}, \quad F_{y}=-\frac{\partial \Phi}{\partial y}, \quad F_{z}=-\frac{\partial \Phi}{\partial z}, \quad F_{z}=-\frac{\partial \Phi}{\partial u}, \\
\frac{\partial^{2} \Phi}{\partial x^{2}}+\frac{\partial^{2} \Phi}{\partial y^{2}}+\frac{\partial^{2} \Phi}{\partial z^{2}}+\frac{\partial^{2} \Phi}{\partial u^{2}}=4 \pi \gamma \nu,
\end{gathered}
$$

where the unit mass is now acted upon by the four-force $\left(F_{x}, F_{y}, F_{z}, F_{u}\right)$.

Simple and natural as this extension was, it ran into a complication once Abraham allowed that the four-force is equal to the four-acceleration $\left(\left(\mathrm{d}^{2} x / \mathrm{d} \tau\right),\left(\mathrm{d}^{2} y / \mathrm{d} \tau\right),\left(\mathrm{d}^{2} z / \mathrm{d} \tau\right),\left(\mathrm{d}^{2} u / \mathrm{d} \tau\right)\right)$ of the unit mass $(\tau$ is the proper time along the trajectory). This equality entailed that the gravitational potential $\Phi$ is related to the speed of light $c$ by

$$
\frac{\mathrm{d} \Phi}{\mathrm{d} \tau}=c \frac{\mathrm{d} c}{\mathrm{~d} \tau}
$$

along the trajectory of a unit mass in free fall. ${ }^{33}$ If the speed of light $c$ were a constant, this result would trivialise Abraham's theory. We would have $0=c(\mathrm{~d} c / \mathrm{d} \tau)=(\mathrm{d} \Phi / \mathrm{d} \tau)$ so that the potential $\Phi$ must be constant along any free fall trajectory of a unit mass.

Abraham's escape was direct if not somewhat opportunistic. He recalled that Einstein had proposed that the speed of light $c$ would no longer be constant in the presence of gravitation but would vary with the potential, basing his new

${ }^{33}$ To see this, note that the line element is $-\mathrm{d} x^{2}-\mathrm{d} y^{2}-\mathrm{d} z^{2}+c^{2} \mathrm{~d} t^{2}=c^{2} \mathrm{~d} \tau^{2}$ and therefore we have

$$
\left(\frac{\mathrm{d} x}{\mathrm{~d} \tau}\right)^{2}+\left(\frac{\mathrm{d} y}{\mathrm{~d} \tau}\right)^{2}+\left(\frac{\mathrm{d} z}{\mathrm{~d} \tau}\right)^{2}+\left(\frac{\mathrm{d} u}{\mathrm{~d} \tau}\right)^{2}=-c^{2} .
$$

Differentiating with respect to $\tau$ we recover

Substituting

$$
\frac{\mathrm{d} x}{\mathrm{~d} \tau} \frac{\mathrm{d}^{2} x}{\mathrm{~d} \tau^{2}}+\frac{\mathrm{d} y}{\mathrm{~d} \tau} \frac{\mathrm{d}^{2} y}{\mathrm{~d} \tau^{2}}+\frac{\mathrm{d} z}{\mathrm{~d} \tau} \frac{\mathrm{d}^{2} z}{\mathrm{~d} \tau^{2}}+\frac{\mathrm{d} u}{\mathrm{~d} \tau} \frac{\mathrm{d}^{2} u}{\mathrm{~d} \tau^{2}}=-c \frac{\mathrm{d} c}{\mathrm{~d} \tau} .
$$

etc. we have

$$
\frac{\mathrm{d}^{2} x}{\mathrm{~d} t^{2}}=F_{x}=-\frac{\partial \Phi}{\partial \tau},
$$

$$
\frac{\mathrm{d} x}{\mathrm{~d} \tau} \cdot \frac{\partial \Phi}{\partial x}+\frac{\mathrm{d} y}{\mathrm{~d} \tau} \cdot \frac{\partial \Phi}{\partial y}+\frac{\mathrm{d} z}{\mathrm{~d} \tau} \cdot \frac{\partial \Phi}{\partial z}+\frac{\mathrm{d} u}{\mathrm{~d} \tau} \cdot \frac{\partial \Phi}{\partial u}=\frac{\mathrm{d} \Phi}{\mathrm{d} \tau}=c \frac{\mathrm{d} c}{\mathrm{~d} \tau}
$$


theory of gravitation upon the proposal. Abraham adopted the same notion, concluding that the potential $\Phi$ would vary directly with $c^{2} .^{34}$

Einstein (1912a, Section 4) protested that this expedient within Abraham's theory precluded the Lorentz transformation holding even infinitesimally. If, following the usual notation, we transform spacetime coordinates $(x, y, z, t)$ to $\left(x^{\prime}, y^{\prime}, z^{\prime}, t^{\prime}\right)$, then the expression for the coordinate differential $\mathrm{d} x^{\prime}$ is

$$
\mathrm{d} x^{\prime}=(\mathrm{d} x-v \mathrm{~d} t) / \sqrt{1-\frac{v^{2}}{c^{2}}}
$$

if the Lorentz transformation is to hold infinitesimally. If this differential is exact so that the infinitesimal $\mathrm{d} x^{\prime}$ can be integrated into a coordinate $x^{\prime}$, then the expression for $\mathrm{d} x^{\prime}$ must satisfy the exactness condition

$$
\frac{\partial}{\partial t}\left\{\frac{1}{\sqrt{1-v^{2} / c^{2}}}\right\}=\frac{\partial}{\partial x}\left\{\frac{-v}{\sqrt{1-v^{2} / c^{2}}}\right\} .
$$

Otherwise the differential transform will not correspond to any coordinate transformation at all. But, Einstein continued, this exactness condition must fail in the case of a static field in which $c$ is a function of the spatial coordinates $x, y$ and $z$ only and if $v$ is constant. For then the expression in curly brackets on the left is independent of $t$, so that the left-hand side vanishes. But the expression in curly brackets on the right will be a function of $x$, so the right-hand side does not vanish.

\title{
Appendix B. Einstein Uses Physical and Formal Mathematical Approaches to Write the Energy Conservation Law
}

\begin{abstract}
The Zurich Notebook contains a successful implementation of Einstein's dual strategy. He generates the conservation law by physical argumentation and then checks that it is a law that could have been found by seeking simple covariant expressions.
\end{abstract}

Early in the deliberations recorded in the Zurich notebook is a page headed 'Gravitation'. (Klein et al., 1995, Doc. 10, p. 10; also control number p. 3006 5R in the duplicate Einstein Archive. This page is represented in facsimile on the dust jacket of Klein et al., 1995.) It commences with the mechanics of a point mass in a gravitational field and concludes with the writing of the law of conservation of energy and momentum, expressed as the vanishing covariant divergence of the stress-energy tensor. The discussion is brief, but discerning its purpose is relatively easy, especially since a similar calculation is given in published form in Einstein and Grossmann (1913, p. 7, pp. 9-11).

\footnotetext{
${ }^{34}$ It was not the only choice. Another alternative was to allow that the mass of the falling body would vary with the gravitational potential. Nordström explored this possibility in his later 1912 theory with the active engagement of Einstein. See Norton (1992, Section 6).
} 
To begin, I shall follow this later development since there Einstein cuts fewer corners.

In the first stage of the calculation (as given in Einstein and Grossmann, 1913, p. 7), Einstein develops the mechanics of a point of mass $m$. He employs a coordinate system $\left(x_{1}, x_{2}, x_{3}, x_{4}=t\right)$ and an invariant line element along the mass' trajectory $\mathrm{ds}^{2}=g_{11} \mathrm{~d} x_{1}^{2}+\cdots+g_{44} \mathrm{~d} x_{4}^{2}$, for $g_{\mu v}$ the metric. The mass in free fall follows a geodesic governed by $\delta \int \mathrm{d} s=0$ which Einstein rewrites as $\delta \int \mathrm{d} s=$ $\delta \int(\mathrm{d} s / \mathrm{d} t) \mathrm{d} t=0=\delta \int H \mathrm{~d} t$, where he has set what we would now call the Lagrangian $H=-m(\mathrm{~d} s / \mathrm{d} t)$. (Beware! In modern texts, the symbol $L$ is used for this quantity.) From the Euler-Lagrange equation associated with this extremal principle,

$$
\frac{\mathrm{d}}{\mathrm{d} t}\left(\frac{\partial H}{\partial \dot{x}_{i}}\right)-\frac{\partial H}{\partial x_{i}}=0
$$

for $i=1,2,3$, Einstein reads off expressions for the force on the mass, $K_{i}=\left(\partial H / \partial x_{i}\right)$ and its momentum $J_{i}=\left(\partial H / \partial \dot{x}_{i}\right)$. (The dot indicates differentiation with respect to $t$.) Following the usual results of Hamiltonian mechanics, the energy is given by

$$
-E=-\sum_{i=1,3} \dot{x}_{i} \frac{\partial H}{\partial \dot{x}_{i}}+H
$$

Recalling that

$$
H=-m \frac{\mathrm{d} s}{\mathrm{~d} t}=-m \sqrt{g_{11} \dot{x}_{1}^{2}+\cdots+g_{44} \dot{x}_{4}^{2}},
$$

Einstein can re-express these quantities as ${ }^{35}$

$$
J_{i}=-m g_{i v} \frac{\mathrm{d} x_{v}}{\mathrm{~d} s}, \quad-E=-m g_{4 v} \frac{\mathrm{d} x_{v}}{\mathrm{~d} s}, \quad K_{i}=-\frac{1}{2} m \frac{\partial g_{\mu v}}{\partial x_{i}} \frac{\mathrm{d} x_{\mu}}{\mathrm{d} s} \frac{\mathrm{d} x_{v}}{\mathrm{~d} t} .
$$

${ }^{35}$ I will not follow Einstein's idiosyncratic notation of 1913. Here summation over repeated indices is implied. Covariant tensorial components are indicated by lowered indices and contravariant components by raised indices. Latin indices range over 1, 2, 3 and Greek indices over 1, 2, 3, 4. The derivation of the quantities in (9) is straightforward as long as one recalls that $x_{i}$ and $\dot{x}_{i}$ are treated as six independent variables in the differentiations and that the $g_{\mu v}$ are functions of $x_{i}$ but not $\dot{x}_{i}$. For example, we have

$$
\frac{\partial H}{\partial \dot{x}_{i}}=\frac{\partial}{\partial \dot{x}_{i}}\left(-m \frac{\mathrm{d} s}{\mathrm{~d} t}\right)=\frac{\partial}{\partial \dot{x}_{i}}\left(-m \sqrt{g_{\mu v} \dot{x}_{\mu} \dot{x}_{v}}\right)=-m \frac{1}{2 \mathrm{~d} s / \mathrm{d} t} 2 g_{i v} \dot{x}_{v}=-m g_{i v} \frac{\mathrm{d} x_{v}}{\mathrm{~d} s}
$$

which gives the expression for $J_{i}$. For $E$ we have

$$
\begin{aligned}
-E & =H-\frac{\partial H}{\partial \dot{x}_{i}} \dot{x}_{i}=-m \frac{\mathrm{d} s}{\mathrm{~d} t}+m g_{i v} \dot{x}_{i} \frac{\mathrm{d} x_{v}}{\mathrm{~d} s}=-m \frac{\mathrm{d} s}{\mathrm{~d} t}\left(1-g_{i v} \frac{\mathrm{d} x_{i}}{\mathrm{~d} s} \frac{\mathrm{d} x_{v}}{\mathrm{~d} s}\right) \\
& =-m \frac{\mathrm{d} s}{\mathrm{~d} t} g_{4 v} \frac{\mathrm{d} x_{4}}{\mathrm{~d} s} \frac{\mathrm{d} x_{v}}{\mathrm{~d} t} \\
& =-m g_{4 v} \frac{\mathrm{d} x_{v}}{\mathrm{~d} s} .
\end{aligned}
$$


The conservation of energy and momentum is a consequence of the EulerLagrange equation (6). In the next phase of the calculation (Einstein and Grossmann, 1913, Part I, pp. 9-11), Einstein extends these conservation laws to a matter distribution. For simplicity he considers the case of a frictionless, pressureless dust cloud with rest mass density $\rho_{0}$. He first converts the expressions (9) for momentum, energy and force into densities, $j_{i}, \eta$ and $k_{i}$. To do this he imagines the mass $m$ of the body to be distributed over a small volume $V$ which he shows to satisfy the relation $\sqrt{-g} V \mathrm{~d} t=V_{0} \mathrm{~d} s$, where $V_{0}$ is the associated rest volume and $g$ the determinant of the metric $g_{\mu v}$. Dividing each expression in (9) by $V$ he recovers ${ }^{36}$

$$
\begin{gathered}
j_{i}=\frac{J_{i}}{V}=-\rho_{0} \sqrt{-g} g_{i v} \frac{\mathrm{d} x_{v}}{\mathrm{~d} s} \frac{\mathrm{d} x_{4}}{\mathrm{~d} s}=-\sqrt{-g} g_{i v} T^{v 4} \\
-\eta=\frac{-E}{V}=-\rho_{0} \sqrt{-g} g_{4 v} \frac{\mathrm{d} x_{v}}{\mathrm{~d} s} \frac{\mathrm{d} x_{4}}{\mathrm{~d} s}=-\sqrt{-g} g_{4 v} T^{v 4} \\
k_{i}=\frac{K_{i}}{V}=-\frac{1}{2} \rho_{0} \sqrt{-g} \frac{\partial g_{\mu v}}{\partial x_{i}} \frac{\mathrm{d} x_{\mu}}{\mathrm{d} s} \frac{\mathrm{d} x_{v}}{\mathrm{~d} t}=-\frac{1}{2} \sqrt{-g} \frac{\partial g_{\mu v}}{\partial x_{i}} T^{\mu v}
\end{gathered}
$$

where the expressions have been simplified by introducing the stress-energy tensor for dust:

$$
T^{\mu v}=\rho_{0} \frac{\mathrm{d} x^{\mu}}{\mathrm{d} s} \frac{\mathrm{d} x^{v}}{\mathrm{~d} s} .
$$

Einstein now proceeds to the final result. He writes down the differential law governing the quantities of $\left(9^{\prime}\right)$ that expresses energy-momentum conservation

$$
\frac{\partial}{\partial x_{v}}\left(\sqrt{-g} g_{\sigma \mu} T^{\mu v}\right)-\frac{1}{2} \sqrt{-g} \frac{\partial g_{\mu v}}{\partial x_{\sigma}} T^{\mu v}=0 .
$$

Exactly how he proceeded from the expressions of ( $\left(9^{\prime}\right)$ to (11) is not so clear and this is a matter of central interest to us. Whatever inferences Einstein used to proceed from $\left(9^{\prime}\right)$ to $(11)$, he seemed to have found them both brief and automatic. In the Zurich Notebook there are no intermediate expressions written down. In Einstein and Grossmann (1913, Part I, p. 10), the jump is made merely by announcing: 'One presumes [vermutet] from the above that the momentum-energy law will have the form [...]' (my emphasis). He does not claim the law has been rigorously deduced.

If (11) is intended to be an automatic translation of the equation of motion (6) into differential form, then considerably more needs to be said to legitimate the inference. For an account of just how much more is needed, see my best

${ }^{36}$ The manipulation is straightforward. For example,

$$
j_{i}=\left(\frac{1}{V}\right) J_{i}=\left(\frac{\sqrt{-g}}{V_{0}} \frac{\mathrm{d} t}{\mathrm{~d} s}\right)\left(-m g_{i v} \frac{\mathrm{d} x_{v}}{\mathrm{~d} s}\right)=-\frac{m}{V_{0}} \sqrt{-g} g_{i v} \frac{\mathrm{d} x_{v}}{\mathrm{~d} s} \frac{\mathrm{d} x_{4}}{\mathrm{~d} s} .
$$


reconstruction in Appendix C. It shows that (6) strongly suggests (11) but that (11) cannot be derived simply by mechanical translation of (6). The brevity of Einstein's remarks suggests that he did not have as elaborate a story in mind. $\mathrm{He}$ may well only have asked how the quantities of $\left(9^{\prime}\right)$ could be combined into a conservation law analogous in form to that used in special relativity. There, the conservation laws were expressed in the four-dimensional formalism in equations like

$$
\frac{\partial T^{\mu v}}{\partial x_{v}}=K^{\mu}
$$

(See Laue's (1911) relativity text, pp. 82, 136.) The divergence of the stress-energy tensor is set equal to the four-force $K^{\mu}$. Equation (11) has this form; its first term is a divergence and its second is a force term. But if one compares (11) and (11') more carefully one sees that the formation of $\left(11^{\prime}\right)$ involves some extrapolation. Only the first three $(i=1,2,3)$ components $K_{i}$ of the four-force density have been identified in the list of $\left(9^{\prime}\right)$. Einstein extrapolates in assuming that the $\sigma=4$ component of the four-force density is analogous in form. Similarly the list does not interpret the stress terms in $T^{\mu v}$ arising in (11), such as $T^{11}$ which, in special relativity, represent normal pressures. Finally, in forming the first divergence term of (11), can we be assured that no other terms in $\sqrt{-g}$ enter? Can we preclude factors of $1 / \sqrt{-g}$, for example?

Einstein had good reason to write down the expression (11). It is the natural expression suggested by the physical considerations. But he also had good reason to hesitate, for the expression might just differ from the correct one in some trivial but nonetheless fatal way. Einstein needed a way to check the result. He could have resorted to the sort of elaborate physical demonstration such as is laid in Appendix C. Instead he chose a much more elegant test. He turned to his mathematical requirements. If all was well, the expression (11) should be generally covariant. In Einstein and Grossmann (1913, Part I, p. 10) Einstein just remarked nonchalantly: 'It turns out in fact that these equations [(11)] are generally covariant under arbitrary substitutions'. A footnote to Grossmann's 'Mathematical Part II' of the paper led readers to the demonstration. The vanishing of (11) turns out, Grossmann showed (Einstein and Grossmann, 1913, Part II, Section 4.1), to be equivalent to the vanishing of the covariant divergence of the stress-energy tensor $T^{\mu v}$.

In the Zurich Notebook, Einstein did not apparently have the luxury of Grossmann's result. Instead he turned to a more indirect test. If (11) was generally covariant, it might just turn out that this general covariance had arisen in a very simple way. The expression in (11) could be conceived as the action on the stress-energy tensor $T^{\mu v}$ by the operator

$$
\frac{\partial}{\partial x_{v}}\left(\sqrt{-g} g_{\sigma \mu} \cdot{ }^{\mu v}\right)-\frac{1}{2} \sqrt{-g} \frac{\partial g_{\mu v}}{\partial x_{\sigma}} \cdot{ }^{\mu v}
$$

where ${ }^{\mu v}$ represents the empty slot into which any second rank, symmetric tensor could be inserted. (It must be symmetric to match the symmetry of $T^{\mu v}$.) 
Equation (11) would be generally covariant if this operator were itself generally covariant; that is, it would yield a generally covariant vector when it acted on a symmetric tensor. It is by no means assured that the operator (12) had this property, even if (11) is generally covariant. In retrospect we can see that (12) is not the unrestricted covariant divergence operator; it had been simplified so that it would form a covariant divergence only when acting on symmetric tensors. That had arisen automatically in the generation of (11). How could Einstein preclude the possibility that some other unrecognised special property of $T^{\mu v}$ had further restricted the operator?

A test was needed and Einstein proceeded with it. He allowed the operator (12) to act on the other second rank, symmetric tensor that was ready to hand, the metric tensor $g_{\mu \nu}$. Einstein wrote the expression for this operator acting on the metric tensor and recorded his expectation ${ }^{37}$

$$
\text { “ } \frac{\partial}{\partial x_{v}}\left(\sqrt{-g} g_{\sigma \mu} g^{\mu \nu}\right)-\frac{1}{2} \sqrt{-g} \frac{\partial g_{\mu v}}{\partial x_{\sigma}} g^{\mu v}=0 \text { or a four-vector". }
$$

Modern readers will recognise that the expression in (13) vanishes identically; it is just equivalent to the vanishing of the covariant divergence of the metric tensor. It is easy on first reading this page to imagine that Einstein is merely reciting a standard identity of the Ricci Levi-Civita calculus. But that is not what Einstein is doing. He does not know if the expression of (13) vanishes or is merely a generally covariant four vector-and his written remark in (13) shows it. Indeed the latter would be all he needed for the operator in (12) to pass his test. Einstein proceeds to evaluate the two terms in (13) and discovers that the expression does indeed vanish. ${ }^{38}$ In effect, by physical reasoning, Einstein has discovered a form of the covariant divergence operator!

Einstein is now assured that (12) has the desired property so that his equation (11) is generally covariant. Indeed it might even then be apparent to Einstein that he could have arrived at (11) by mathematical reasoning as well. If, instead of pursuing physical considerations, he had merely asked 'what simple, generally covariant divergence operators are there', he might well have been led directly to

${ }^{37}$ In the quote I have silently modernised Einstein's notation as indicated above.

${ }^{38}$ Einstein finds the first term is

$$
\frac{\partial}{\partial x_{v}}\left(\sqrt{-g} g_{\sigma \mu} g^{\mu \nu}\right)=\frac{\partial}{\partial x_{v}}\left(\sqrt{-g} \delta_{\sigma}^{v}\right)=\frac{\partial}{\partial x_{v}}(\sqrt{-g}) .
$$

Since

$$
\frac{1}{g} \frac{\partial g}{\partial x_{\sigma}}=\frac{\partial g_{\mu v}}{\partial x_{\sigma}} g^{\mu v}
$$

this first term is equal to the negation of the second term:

$$
\frac{1}{2} \sqrt{-g} \frac{\partial g_{\mu v}}{\partial x_{\sigma}} g^{\mu v}=\frac{1}{2} \sqrt{-g} \frac{1}{g} \frac{\partial g}{\partial x_{\sigma}}=\frac{1}{2} \frac{1}{\sqrt{-g}} \frac{\partial(-g)}{\partial x_{\sigma}}=\frac{\partial}{\partial x_{\sigma}}(\sqrt{-g}) .
$$


(12) and thence (11). In any case, his physical argumentation had produced a result that matched his mathematical expectations. He wrote across the bottom of the page 'Stimmt' ['Correct'].

A small complication in the calculation shows that Einstein's caution in checking the result was not misplaced. As Grossmann showed, (12) is not the covariant divergence operation after all. It lacks a multiplicative factor of $1 / \sqrt{-g}$. This turns out not to compromise Einstein's overall result. Equation (11) still vanishes in all coordinate systems whether or not this extra factor is included. But it does mean that Einstein was incorrect in identifying (13) as a four-vector and in his marginal remark written next to equation (11): 'In general a corresponding vector'. The operations involved in the formation of (11) produce a vector from a second rank, symmetric tensor $T^{\mu v}$ only if the missing factor of $1 / \sqrt{-g}$ is included. As it turns out, what saves Einstein is the fact that both (11) and (13) are set to zero so the effect of the factor is eliminated; the equations are equivalent and thus both versions are generally covariant. However were circumstances to arise in which (11) did not vanish, then the difference might matter.

\section{Appendix C. The Missing Derivation}

To proceed from the mechanics of a point mass to the laws governing distributed matter, Einstein would need an argument augmented with an appeal to naturalness or with further material assumptions.

The conservation law (11) can almost be recovered from an argument that takes the corresponding laws of the mechanics of a point mass and translates them into equations governing distributed matter. The final step requires either an appeal to naturalness or a material augmentation of the argument. There is no assurance that Einstein would have offered this sort of derivation if it were requested. However it is plausible that he would. After reporting the covariance properties of (11) in Einstein and Grossmann (1913, Part I, p. 10), he proceeded to observe: 'Further, the equations of motion of a material point, from which we began, can be derived again by integration over the current threads'. The derivation below amounts to Einstein's proposed rederivation conducted in reverse. We can also specify more precisely just how Einstein's proposed rederivation of the equations of motion would have proceeded by consulting the analogous derivation that Einstein sketches within special relativity in his text (Einstein, 1922, pp. 52-53). Although the derivation from the distributed law (11) to the equation of motion (6) is straight forward, its reversal here is not.

The Euler-Lagrange equation (6) expresses momentum conservation for the point mass. It will translate into the $\sigma=1,2,3$ components of equation (11), which express momentum conservation. To recover them we represent the mass point as a small, extended body, as shown in Fig. 1. 


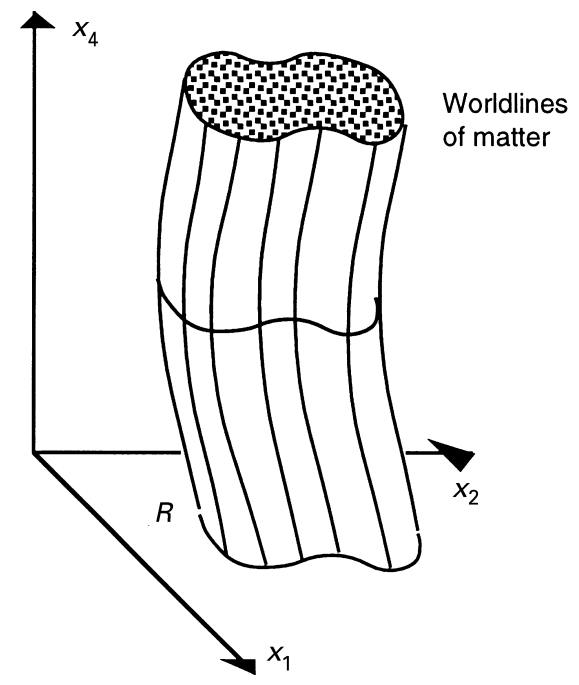

Fig. 1. An isolated mass

The stress-energy tensor for the matter vanishes everywhere except within the small region $R$ of each spacelike surface of constant $t=x_{4}$ occupied by the mass. Expressed in terms of the quantities of (9), the Euler-Lagrange equation (6) adopts the familiar form

$$
K_{i}=\frac{\mathrm{d} J_{i}}{\mathrm{~d} t}
$$

Re-expressing these quantities in terms of densities pertinent to the mass as portrayed in Fig. 1 we have

$$
K_{i}=\int_{\substack{\text { all } \\ \text { space }}} k_{i} \mathrm{~d} x_{1} \mathrm{~d} x_{2} \mathrm{~d} x_{3}=\int_{\substack{\text { space } \\ \text { space }}}\left(-\frac{1}{2} \sqrt{-g} \frac{\partial g_{\mu \nu}}{\partial x_{i}} T^{\mu v}\right) \mathrm{d} x_{1} \mathrm{~d} x_{2} \mathrm{~d} x_{3},
$$

where I have substituted for $k_{i}$ from $\left(9^{\prime}\right)$. We also have

$$
\begin{aligned}
\frac{\mathrm{d} J_{i}}{\mathrm{~d} t}=\frac{\mathrm{d}}{\mathrm{d} t} \int_{\substack{\text { all } \\
\text { space }}} j_{i} \mathrm{~d} x_{1} \mathrm{~d} x_{2} \mathrm{~d} x_{3} & =\frac{\mathrm{d}}{\mathrm{d} t} \int_{\substack{\text { space } \\
\text { all }}}\left(-\sqrt{-g} g_{i v} T^{v 4}\right) \mathrm{d} x_{1} \mathrm{~d} x_{2} \mathrm{~d} x_{3} \\
& =\int_{\substack{\text { space } \\
\text { all }}} \frac{\partial}{\partial t}\left(-\sqrt{-g} g_{i v} T^{v 4}\right) \mathrm{d} x_{1} \mathrm{~d} x_{2} \mathrm{~d} x_{3} .
\end{aligned}
$$

The last equality requires that we choose a particular coordinate system in which curves of constant $x_{1}, x_{2}$ and $x_{3}$ coincide with the trajectories of the 
elements of the mass. In that case we have

$$
\frac{\mathrm{d}}{\mathrm{d} t}=\frac{\partial}{\partial t}+\frac{\mathrm{d} x_{k}}{\mathrm{~d} t} \frac{\partial}{\partial x_{k}}=\frac{\partial}{\partial t}
$$

since $\mathrm{d} x_{k} / \mathrm{d} t=0$. The results arrived at will therefore be restricted to these special coordinate systems. A more elaborate derivation could avoid this restriction, but it is not needed since, as Grossmann showed, the final result is generally covariant and thus holds in all coordinate systems.

To proceed we apply Gauss' theorem in the three-space spanned by coordinates $x_{1}, x_{2}$ and $x_{3}$ at some fixed $x_{4}$ to infer that

$$
\int_{\substack{\text { sall } \\ \text { space }}} \frac{\partial}{\partial x_{k}}\left(-\sqrt{-g} g_{i \mu} T^{\mu k}\right) \mathrm{d} x_{1} \mathrm{~d} x_{2} \mathrm{~d} x_{3}=0,
$$

where summation over $k$ extends to just $k=1,2,3$. This integrated divergence vanishes since Gauss' theorem allows us to equate it with a surface integral that lies outside $R$ so that $T^{\mu v}=0$ everywhere on the surface. Since (17) vanishes, we can add it to the expression for $\mathrm{d} J_{i} / \mathrm{d} t$ and recover

$$
\frac{\mathrm{d} J_{i}}{\mathrm{~d} t}=\int_{\substack{\text { all } \\ \text { space }}} \frac{\partial}{\partial x_{v}}\left(-\sqrt{-g} g_{i \mu} T^{\mu v}\right) \mathrm{d} x_{1} \mathrm{~d} x_{2} \mathrm{~d} x_{3} .
$$

So equation $\left(6^{\prime}\right)$ is now equivalent to

$$
\begin{aligned}
& \int_{\substack{\text { sll } \\
\text { space }}} \frac{\partial}{\partial x_{v}}\left(-\sqrt{-g} g_{i \mu} T^{\mu v}\right) \mathrm{d} x_{1} \mathrm{~d} x_{2} \mathrm{~d} x_{3} \\
= & \int_{\substack{\text { all } \\
\text { space }}}\left(-\frac{1}{2} \sqrt{-g} \frac{\partial g_{\mu v}}{\partial x_{i}} T^{\mu v}\right) \mathrm{d} x_{1} \mathrm{~d} x_{2} \mathrm{~d} x_{3} .
\end{aligned}
$$

A sufficient condition for $\left(6^{\prime}\right)$ to hold is the equality of the terms within the scope of the integration operations

$$
\frac{\partial}{\partial x_{v}}\left(\sqrt{-g} g_{i \mu} T^{\mu v}\right)=\frac{1}{2} \sqrt{-g} \frac{\partial g_{\mu v}}{\partial x_{i}} T^{\mu v},
$$

which is just the $\sigma=1,2,3$ components of the result (11) sought. For the derivation to be complete, we would need to show that the result is also necessary for ( $\left.6^{\prime}\right)$; that is, that $\left(6^{\prime}\right)$ entails $(11 ; \sigma=1,2,3)$. But this last entailment does not obtain. Equation $\left(6^{\prime}\right)$ can be recovered from alternatives to $(11 ; \sigma=1,2,3)$. In the argument above, we could add a three-space divergence $\left(\partial / \partial x_{k}\right)\left(-\sqrt{-g} g_{i \mu} T^{\mu k}\right)$ in $T^{\mu v}$ to the integral expression for $\mathrm{d} J_{i} / \mathrm{d} t$ exactly because this divergence would vanish by Gauss' theorem in the integration. So variants of $(11 ; \sigma=1,2,3)$ with other such divergence terms added to the left hand side are compatible with $\left(6^{\prime}\right)$. 
Most simply we might just neglect to add the above mentioned divergence term, so that in place of $(11 ; \sigma=1,2,3)$ we would have

$$
\frac{\partial}{\partial t}\left(\sqrt{-g} g_{i \mu} T^{\mu 4}\right)=\frac{1}{2} \sqrt{-g} \frac{\partial g_{\mu v}}{\partial x_{i}} T^{\mu v}
$$

but we can still recover $\left(6^{\prime}\right)$ from it by integration.

Needless to say, the derivation could be completed, but only by adding new assumptions that go beyond the original enterprise of translating $\left(6^{\prime}\right)$ into differential form. We might note that the modified forms of $(11 ; \sigma=1,2,3)$ are not likely to give generally covariant expressions. But that now invokes Einstein's mathematical argument. Or we might note that analogy with special relativity requires symmetry over all four coordinates in the derivative term on the left of $(11 ; \sigma=1,2,3)$. But then we would need to argue that $(11 ; \sigma=1,2,3)$ is the only way to achieve this symmetry. Or we might wonder if a variant of (11; $\sigma=1,2,3)$ will still yield the correct special relativistic and Newtonian limit. But it will if $(11 ; \sigma=1,2,3)$ is augmented by cleverly contrived terms that vanish in these limiting processes. Finally we might note that the divergence on the left hand side of $(11 ; \sigma=1,2,3)$ is needed if the resulting law is to express a conservation of momentum upon integration in other cases of distributed matter in which the stress-energy tensor does not vanish on the spatial boundary of the region considered. That is true, but it is now an extension of the original argument. We require that our differential law return both the conservation of momentum for a point mass and also for other cases as well (and it will need physical interpretation of other terms of $T^{\mu v}$ ).

To proceed with the derivation, we also need to recover the $\sigma=4$ component of (11) and here again we discover incompleteness in the physical argument Einstein sketches. We might expect to be forced to the $\sigma=4$ component of (11) once we have the $\sigma=1,2,3$ components of (11), if (11) is to be generally covariant - but that is a mathematical argument and not the physical derivation sought. The latter derivation requires that we begin with an equation that expresses energy conservation for the point mass. Such an equation was not written by Einstein in his developments. The relevant equation requires an expression for $P$, the power developed. It is recovered from the familiar result in Hamiltonian mechanics that ${ }^{39}(\mathrm{~d} E / \mathrm{d} t)=-(\partial H / \partial t)$. Substituting for $H$, the

${ }^{39}$ This result is a consequence of the Euler-Lagrange equation (6). It is recovered by regrouping terms in

$$
\frac{\mathrm{d} H}{\mathrm{~d} t}=\frac{\partial H}{\partial t}+\frac{\partial H}{\partial \dot{x}_{i}} \frac{\mathrm{d} \dot{x}_{i}}{\mathrm{~d} t}+\frac{\partial H}{\partial x_{i}} x_{i}=\frac{\partial H}{\partial t}+\frac{\partial H}{\partial \dot{x}_{i}} \frac{\mathrm{d} \dot{x}_{i}}{\mathrm{~d} t}+\frac{\mathrm{d}}{\mathrm{d} t}\left(\frac{\partial H}{\partial \dot{x}_{i}}\right) \dot{x}_{i}=\frac{\partial H}{\partial t}+\frac{\mathrm{d}}{\mathrm{d} t}\left(\frac{\partial H}{\partial \dot{x}_{i}} \dot{x}_{i}\right),
$$

recalling that

$$
E=\frac{\partial H}{\partial \dot{x}_{i}} \dot{x}_{i}-H
$$


expression for power turns out to be

$$
P=\frac{\mathrm{d} E}{\mathrm{~d} t}=\frac{1}{2} m \frac{\partial g_{\mu v}}{\partial x_{4}} \frac{\mathrm{d} x_{\mu}}{\mathrm{d} s} \frac{\mathrm{d} x_{v}}{\mathrm{~d} t} .
$$

Forming densities as before, we have the power density

$$
p=\frac{P}{V}=\frac{1}{2} \rho_{0} \sqrt{-g} \frac{\partial g_{\mu v}}{\partial x_{4}} \frac{\mathrm{d} x_{\mu}}{\mathrm{d} s} \frac{\mathrm{d} x_{v}}{\mathrm{~d} s} .
$$

The equation that expresses energy conservation is

$$
\frac{\mathrm{d} E}{\mathrm{~d} t}=P .
$$

This is reexpressed in terms of the energy and power densities $\eta$ and $p$ for the mass in Fig. 1 as

$$
\frac{\mathrm{d}}{\mathrm{d} t} \int_{\substack{\text { all } \\ \text { space }}} \eta \mathrm{d} x_{1} \mathrm{~d} x_{2} \mathrm{~d} x_{3}=\int_{\substack{\text { sall } \\ \text { space }}} p \mathrm{~d} x_{1} \mathrm{~d} x_{2} \mathrm{~d} x_{3} .
$$

We now substitute the above expression for $p$ and the earlier expression $\left(9^{\prime}\right)$ for $\eta$. Proceeding as before in the coordinate system in which $\mathrm{d} / \mathrm{d} t=\partial / \partial t$ and with addition of a term analogous to (17) above, equation (22) is equivalent to

$$
\begin{gathered}
\int_{\substack{\text { all } \\
\text { space }}} \frac{\partial}{\partial x_{v}}\left(\sqrt{-g} g_{4 \mu} T^{\mu v}\right) \mathrm{d} x_{1} \mathrm{~d} x_{2} \mathrm{~d} x_{3} \\
=\int_{\substack{\text { all } \\
\text { space }}}\left(\frac{1}{2} \rho_{0} \sqrt{-g} \frac{\partial g_{\mu v}}{\partial x_{4}} \frac{\mathrm{d} x_{\mu}}{\mathrm{d} s} \frac{\mathrm{d} x_{v}}{\mathrm{~d} s}\right) \mathrm{d} x_{1} \mathrm{~d} x_{2} \mathrm{~d} x_{3} .
\end{gathered}
$$

As before, $\left(22^{\prime}\right)$ will hold if

$$
\frac{\partial}{\partial x_{v}}\left(\sqrt{-g} g_{4 \mu} T^{\mu v}\right)=\frac{1}{2} \rho_{0} \sqrt{-g} \frac{\partial g_{\mu v}}{\partial x_{4}} \frac{\mathrm{d} x_{\mu}}{\mathrm{d} s} \frac{\mathrm{d} x_{v}}{\mathrm{~d} s}
$$

which is the $\sigma=4$ component of (11) desired. Again, further assumption is needed if we are to deduce this last result from (22). The argument leading to (11; $\sigma=1,2,3)$ could not preclude an additional three space divergence term. The same problem arises with the $\sigma=4$ component.

\section{Appendix D. A Brief Critique of Einstein's Viewpoint}

That many successful, physical theories find simple mathematical formulations might equally reflect the malleability of existing formalisms and the ingenuity of mathematicians in creating new ones to fit the theory at hand. 
Einstein makes two claims in his 1933 Herbert Spenser lecture. First is a claim about the world: 'Nature is the realisation of the simplest conceivable mathematical ideas'. The second is about successful methods and is a consequence of the first: we can find the true theories by looking for those that are mathematically simple. I shall express doubts about both claims here.

Einstein's evidence for the first claim is that so many of our successful theories do in fact have simple mathematical expression. This evidence does not compel acceptance of Einstein's claim. It has most persuasive power if we presume that our mathematical formalism is fixed. The true physical laws are those that find simple expression in this fixed formalism. But formalisms are not fixed. They evolve according to the practical needs of the mathematicians and their customers. The more common scenario is that a new physical theory emerges, often in cumbersome mathematical clothing, and a mathematical formalism is refined and simplified by mathematicians in order to accommodate the theory. As a result, the theory automatically finds simple expression in that formalism-not because nature is built from a simple mathematical blueprint, but because we have contrived mightily to find a formalism in which the theory is simple.

This is just what happened with the emergence of quantum mechanics. It struggled through a quarter century as the old quantum theory in which both classical concepts and formalism were strained to contain it. With the breakthrough to the new quantum theory starting in 1925, several new formal approaches emerged: Heisenberg's matrices, Schrödinger's waves and Dirac's q-numbers. It was only after considerable further mathematical work that a natural formal home for the theory was found in terms of linear operators on Hilbert spaces. This example also reveals a vagueness in Einstein's claim. A physical theory will find simple expression only if the right formalism is selected. Given the huge range of choices and the possibility of generating more formalisms should none do the job, one must be less impressed when this or that theory eventually finds simple mathematical expression.

In this regard, general relativity appears exceptional. The right mathematical formalism lay waiting, presciently, in the mathematical literature. But this last assessment is somewhat over-optimistic. The Ricci Levi-Civita system of 1901 was still not a perfect match for Einstein's theory. As a result of the enormous interest generated by the success of Einstein's theory, there was an explosion of new mathematical work, all designed to enhance Einstein's theory. One of the most important was Levi-Civita's introduction of the notion of parallel displacement in 1917, after general relativity had been completed.

Finally we should guard against a simple selection effect. Imagine that some true theories can be expressed simply in a mathematical formalism ready to hand and that others cannot. In this scenario we are most likely to find the first type of theory and less likely to find the second. So the fact that our physics texts are bursting with theories in simple mathematical clothing would just reflect our inability to discover the ones that require complicated expression.

What of Einstein's second claim? Perhaps nature is not constructed from a mathematical blueprint. Does that still preclude the heuristic value of 
assuming that it does? We might wonder if a false presumption could be of sustained heuristic power. How could it consistently license action that fits the world if nothing in the presumption matches the world? We should also recall the hidden catch in the heuristic. It can only succeed if we happen to choose the right formalism in which to write our simple equations. As long as we seek simple laws in the formalism of classical phase spaces, we are unlikely to write down quantum results. Nonetheless, the more immediate test lies in checking whether the heuristic has historically had power. Here the results are mixed. Einstein clearly takes the success with general relativity as a premiere illustration. But one success does not distinguish between a flawed method that was lucky once and a good method that will enjoy repeated success. Further successful applications are needed. They would have been supplied, had Einstein's quest for a unified field theory met with acclaimed success. But the physics community has voted with their pencils. Einstein's theory and his programme languish in neglect.

We should not marvel at the hand because it fits the glove.

\section{References}

Abraham, M. (1912) 'Zur Theorie der Gravitation', Physikalische Zeitschrift 13, $1-4$.

Beck, A. (1987) (translator) The Collected Papers of Albert Einstein. Volume 1: The Early Years, 1879-1902 (P. Havas, consultant) (Princeton: Princeton University Press).

Beck, A. (1995) (translator) The Collected Papers of Albert Einstein. Volume 5: The Swiss Years: Correspondence, 1902-1914 (D. Howard, consultant) (Princeton: Princeton University Press).

Corry, L. (1999) 'David Hilbert between Mechanical and Electromagnetic Reductionism', Archive for History of Exact Sciences 53, 489-527.

Corry, L., Renn, J. and Stachel, J. (1997) 'Belated Decision in the Hilbert-Einstein Priority Dispute', Science 278, 1270-1273.

Earman, J. and Glymour, C. (1978) 'Einstein and Hilbert: Two Months in the History of General Relativity', Archive for History of Exact Sciences 19, 291-308.

Earman, J. and Janssen, M. (1993) 'Einstein's Explanation of the Motion of Mercury's Perihelion', in Earman, Janssen and Norton (eds) (1993), pp. 129-172.

Earman, J., Janssen, M. and Norton, J. D. (eds) (1993) The Attraction of Gravitation: New Studies in the History of General Relativity: Einstein Studies, Volume 5 (Boston: Birkhäuser).

Einstein, A. (1912a) 'Lichtgeschwindigkeit und Statik des Gravitationsfeldes', Annalen der Physik 38, 355-369.

Einstein, A. (1912b) 'Bemerkung zu Abrahams vorangehender Auseinandersetzung "Nochmals Relativität und Gravitation", Annalen der Physik 39, 704.

Einstein, A. (1914) 'Die formale Grundlage der allgemeinen Relativitätstheorie', Königlich Preussische Akademie der Wissenschaften (Berlin). Sitzungsberichte, 1030-1085.

Einstein, A. (1915a) 'Zur allgemeinen Relativitätstheorie', Königlich Preussische Akademie der Wissenschaften (Berlin). Sitzungsberichte, 778-786 (submitted 4 November 1915).

Einstein, A. (1915b) 'Zur allgemeinen Relativitätstheorie (Nachtrag)', Königlich Preussische Akademie der Wissenschaften (Berlin). Sitzungsberichte, 799-801 (submitted 11 November 1915). 
Einstein, A. (1915c) 'Erklärung der Perihelbewegung des Merkur aus der allgemeinen Relativitätstheorie', Königlich Preussische Akademie der Wissenschaften (Berlin). Sitzungsberichte, 831-839 (submitted 18 November 1915).

Einstein, A. (1915d) 'Die Feldgleichungen der Gravitation', Königlich Preussische Akademie der Wissenschaften (Berlin). Sitzungsberichte, 844-847 (communicated 25 November 1915, submitted 26 November 1915).

Einstein, A. (1918a) 'Principles of Research', in Einstein (1954), pp. 224-227.

Einstein, A. (1918b) 'Nachtrag [to Weyl, 1918]', Königlich Preussische Akademie der Wissenschaften (Berlin). Sitzungsberichte, 478.

Einstein, A. (1922) The Meaning of Relativity (Princeton: Princeton University Press, 1974, 5 th edn).

Einstein, A. (1933a) 'On the Methods of Theoretical Physics', in Einstein (1954), pp. 270-276.

Einstein, A. (1933b) 'Notes on the Origin of the General Theory of Relativity', in Einstein (1954), pp. 285-290.

Einstein, A. (1936) 'Physics and Reality', in Einstein (1954), pp. 290-323.

Einstein, A. (1949) Autobiographical Notes, P. A. Schillp (translator) (La Salle and Chicago: Open Court, 1979, Centennial Edition).

Einstein, A. (1954) Ideas and Opinions (New York: Bonanza).

Einstein, A. and Grossmann, M. (1913) Entwurf einer verallgemeinerten Relativitätstheorie und einer Theorie der Gravitation (Leipzig: B. G. Teubner), Parts I and II, separatum; with addendum by Einstein in Zeitschrift für Mathematik und Physik, 63 (1914), pp. 225-261.

Fölsing, A. (1997) Albert Einstein: A Biography, E. Osers (translator) (New York: Viking).

Frank, P. (1947) Einstein: His Life and Times, G. Rosen (translator). Edited and revised by Shuichi Kusaka (New York: Alfred A. Knopf).

Goenner, H., Renn, J., Ritter, J. and Sauer, T. (eds), (1999) The Expanding Worlds of General Relativity: Einstein Studies, Volume 7 (Boston: Birkhäuser).

Hentschel, A. M. (1998) (translator) The Collected Papers of Albert Einstein. Volume 8: The Berlin Years: Correspondence, 1914-1918 (K. Hentschel, consultant) (Princeton: Princeton University Press).

Hilbert, D. (1915) 'Die Grundlagen der Physik (Erste Mitteilung)', Königliche Gesellschaft der Wissenschaften zu Göttingen. Mathematisch-physikalische Klasse. Nachrichten, 395-407.

Howard. D. and Norton J. D. (1993) 'Out of the Labyrinth? Einstein, Hertz and the Göttingen Answer to the Hole Argument', in Earman, Janssen and Norton (eds) (1993), pp. 30-62.

Howard, D. and Stachel, J. (eds) (1989) Einstein and the History of General Relativity: Einstein Studies, Vol. 1 (Boston: Birkhäuser).

Janssen, M. (1999) 'Rotation as the Nemesis of Einstein's Entwurf Theory', in H. Goenner et al. (eds), pp. 127-157.

Klein, M. J., Kox, A. J. and Schulmann, R. (eds) (1993) The Collected Papers of Albert Einstein. Volume 5: The Swiss Years: Correspondence, 1902-1914 (Princeton: Princeton University Press).

Klein, M. J., Kox, A. J., Renn, J. and Schulmann, R. (eds) (1995) The Collected Papers of Albert Einstein. Volume 4: The Swiss Years: Writings, 1912-1914 (Princeton: Princeton University Press).

Laue, M. (1911) Das Relativitätsprinzip (Braunschweig: Vieweg \& Sohn).

Mehra, J. (1974) Einstein, Hilbert and the Theory of Gravitation (Dordrecht: D. Reidel).

McCormmach, R. (1976) 'Editor's Foreword', Historical Studies in the Physical Sciences 7 (Princeton: Princeton University Press), pp. xi-xxxv.

Michelmore, P. (1962) Einstein, Profile of the Man (London). 
Minkowski, H. (1908) 'Space and Time', in H. A. Lorentz et al., The Principle of Relativity (New York: Dover, 1952), pp. 73-96.

Norton, J. D. (1984) 'How Einstein found his Field Equations: 1912-1915', Historical Studies in the Physical Sciences 14, 253-316; reprinted in Howard and Stachel (eds) (1989), pp. 101-159.

Norton, J. D. (1985) 'What was Einstein's Principle of Equivalence?', Studies in History and Philosophy of Science, 16, 203-246; reprinted in Howard and Stachel (eds) (1989), pp. 5-47.

Norton, J. D. (1992) 'Einstein, Nordström and the Early Demise of Scalar, LorentzCovariant Theories of Gravitation', Archive for the History of Exact Sciences 45, 17-94.

Norton, J. D. (1993) 'General Covariance and the Foundations of General Relativity: Eight Decades of Dispute', Reports on Progress in Physics 56, 791-858.

Norton, J. D. (1995) 'Eliminative Induction as a Method of Discovery: Einstein's Discovery of General Relativity', in J. Leplin (ed.), The Creation of Ideas in Physics: Studies for a Methodology of Theory Construction (Dordrecht: Kluwer), pp. 29-69.

Norton, J. D. (manuscript a) 'A Conjecture on Einstein, the Reality of Spacetime Coordinate Systems and the Disaster of 1913'.

Norton, J. D. (manuscript b) 'An Elementary Exposition of Einstein's Errors of 1913'.

Pais, A. (1982) Subtle is the Lord: The Science and Life of Albert Einstein (Oxford: Clarendon).

Reid, C. (1986) Hilbert-Courant (New York: Springer-Verlag).

Renn, J. and Sauer, T. (1999) 'Heuristics and Mathematical Representation in Einstein's Search for a Gravitational Field Equation', in H. Goenner et al. (eds) (1999), pp. $87-125$.

Renn, J. and Stachel, J. (1999) 'Hilbert's Foundation of Physics: From a Theory of Everything to a Constituent of General Relativity', Max Planck Institute for the History of Science, Berlin, preprint 118.

Ricci, G. and Levi-Civita, T. (1901) 'Méthodes de Calcul Différentiel Absolu et leurs Application', Mathematische Annalen 54, 125-201; reprinted in T. Levi-Civita (1954) Opere Matematiche, Vol. 1 (Bologna), pp. 479-559.

Sauer, T. (1999) 'The Relativity of Discovery: Hilbert's First Note on the Foundations of Physics', Archive for History of Exact Sciences 53, 529-575.

Schulmann, R., Kox, A. J., Janssen, M. and Illy, J. (eds) (1998) The Collected Papers of Albert Einstein. Volume 8: The Berlin Years: Correspondence, 1914-1918. Part A: 1914-1917, Part B: 1918 (Princeton: Princeton University Press).

Seelig, C. (1956) Albert Einstein: A Documentary Biography, translated by M. Savill (London: Staples Press).

Smith, F. W. F. (1962) The Professor and the Prime Minister (Boston).

Sommerfeld, A. (1910) 'Zur Relativitätstheorie. I: Vierdimensionale Vektoralgebra. II: Vierdimensionales Vektoranalysis', Annalen der Physik 32, pp. 749-776; 33, pp. 649-689.

Stachel, J., Cassidy, D. C., Schulmann, R. and Renn, J. (eds) (1987) The Collected Papers of Albert Einstein. Volume 1: The Early Years, 1879-1902 (Princeton: Princeton University Press).

Straus, E. G. (1982) 'Reminiscences', in G. Holton and Y. Elkana (eds), Albert Einstein: Historical and Cultural Perspectives (Princeton: Princeton University Press), pp. 417-423.

Weyl, H. (1918) 'Gravitation und Elektrizität', Königlich Preussische Akademie der Wissenschaften (Berlin). Sitzungsberichte, 465-478,478-480. 